\title{
Single-electron source: Adiabatic versus nonadiabatic emission
}

\author{
Michael Moskalets, ${ }^{1,2}$ Géraldine Haack, ${ }^{1,3}$ and Markus Büttiker ${ }^{1}$ \\ ${ }^{1}$ Département de Physique Théorique, Université de Genève, CH-1211 Genève 4, Switzerland \\ ${ }^{2}$ Department of Metal and Semiconductor Physics, NTU “Kharkiv Polytechnic Institute," 61002 Kharkiv, Ukraine \\ ${ }^{3}$ Dahlem Center for Quantum Complex Systems and Fachbereich Physik, Freie Universität Berlin, 14195 Berlin, Germany
}

(Received 24 January 2013; revised manuscript received 7 March 2013; published 29 March 2013)

\begin{abstract}
We investigate adiabatic and nonadiabatic emission of single particles into an edge state using an analytically solvable dynamical scattering matrix model of an on-demand source. We compare adiabatic and nonadiabatic emissions by considering two geometries: a collider geometry where two emitters are coupled to two different edge states and a series geometry where two emitters are coupled to the same edge state. Most effects observed for adiabatic emitters also occur for nonadiabatic emitters. In particular this applies to effects arising due to the overlap of wave packets colliding at a quantum point contact. Specifically we compare the Pauli peak (the fermionic analog of the bosonic Hong-Ou-Mandel dip) for the adiabatic and nonadiabatic collider and find them to be similar. In contrast we find a striking difference between the two operating conditions in the series geometry in which particles are emitted into the same edge state. Whereas the squared average charge current can be nullified for both operating conditions, the heat current can be made to vanish only with adiabatic emitters.
\end{abstract}

DOI: 10.1103/PhysRevB.87.125429

PACS number(s): 72.10.-d, 73.23.-b, 73.50.Td, 73.22.Dj

\section{INTRODUCTION}

Recent progress in the field of dynamical quantum transport ${ }^{1}$ opens new and fascinating perspectives for exploring and understanding mesoscopic and nanoscopic conductors. With the implementation of an on-demand single-electron emitter ${ }^{2,3}$ not relying on electron-electron interaction it is possible to address directly dynamic properties of a single-electron state in solids. The single-particle nature of emitted wave packets was demonstrated using the noise measurements. ${ }^{47}$ To investigate the coherence properties of emitted wave packets an approach based on the measurement of current correlations at a beam splitter ${ }^{8,9}$ and an approach based on the measurement of current at the output of an interferometer ${ }^{10}$ have already been proposed.

The state of an electron depends crucially on the way it is emitted; see Fig. 1. In most experiments with such high-speed single-electron sources-see, e.g., Refs. 11-15, also the theoretical proposal in Ref. 16 and the analysis of a single-electron capture in Ref. 17-electrons are emitted from the quantum dot with energy far above the Fermi level. On the other hand, in theory many effects were predicted for electrons emitted adiabatically almost at the surface of the Fermi sea: the shot-noise quantization; ${ }^{18-21}$ the shot-noise suppression effect; ${ }^{18,22}$ a two-particle interference and entanglement generation ${ }^{23-25}$ interesting for quantum information applications; ${ }^{26}$ a particle reabsorption; ${ }^{27,28}$ the suppression of a single-particle interference by collisions. ${ }^{29}$ Recently also single and few-electron sources based on the generation of Lorentzian voltage pulses applied to a ballistic conductor as proposed in Refs. 30 and 31, and discussed in detail in Ref. 32, have now been realized experimentally in Ref. 33 . The properties of a single-electron state generated by such a source are similar to those of the state emitted adiabatically by a single lead mesoscopic capacitor. ${ }^{2}$

Our aim here is to answer the question of whether one can expect similar effects with particles emitted nonadiabatically or not. For this purpose we analyze the single-electron source of Ref. 2 because it can operate in both adiabatic and

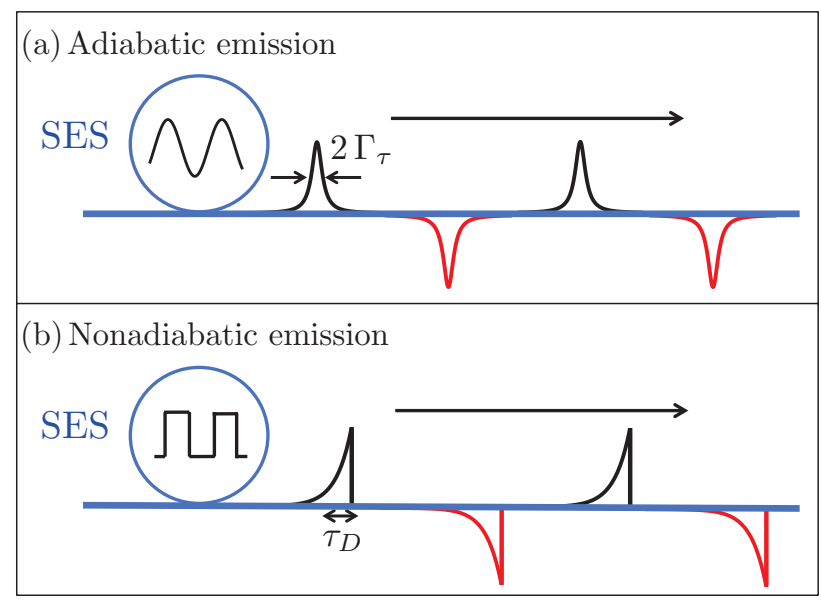

FIG. 1. (Color online) Single-electron sources (SES) emit a train of electrons (black pulses) alternating with holes (red pulses) into an edge states (serving as an electronic waveguide). Electron and hole pulses are well separated in time and space. The shape of the single-particle wave packets depends crucially on the way the source is driven: (a) Adiabatic emission: the SES is driven by a smooth periodic potential, the pulse as a function of time has a Lorentzian shape with width $2 \Gamma_{\tau}$. (b) Nonadiabatic emission: the SES is driven by a pulsed periodic potential, the pulse as a function of time has an exponential shape characterized by the dwell time $\tau_{D}$.

nonadiabatic emission conditions. Moreover it seems that its properties are well described by a noninteracting theory. $2,34,35$ That makes it possible to develop a relatively simple analytical theory which describes both operating conditions.

We use a noninteracting model, $, 36,37$ in which the source consists of a single circular edge state, a Fabry-Pérot cavity, weakly coupled to a linear edge state, which plays the role of an electron waveguide. This analytical model is in good agreement with actual experiments. In the weak-coupling limit, the transparency of the quantum point contact connecting the cavity and the electron waveguide is small, $T \ll 1$. All relevant energies are smaller than the Fermi energy $\mu$ and the 
energy spectrum of electrons can be linearized in the vicinity of $\mu$. That results in the equidistant spectrum of the cavity with level spacing $\Delta=h / \tau$ defined by the time of flight $\tau$ around the circular edge state of the cavity. A metallic top gate with potential $U(t)$ periodically changes the position of the quantum levels in the cavity. We assume optimal operating conditions $^{2,5,35}$ which require that the Fermi level is positioned in the middle of two levels of the cavity and the gate potential changes with amplitude $\Delta$. In this case only one level crosses the Fermi energy: When it raises above the Fermi level an electron is emitted from the cavity into the waveguide, whereas when the level sinks below the Fermi level an electron is absorbed by the cavity hence a hole appears in the stream of electrons within the waveguide. Such a source generates no dc current and is often referred to as a quantum capacitor. ${ }^{34,38}$

To get an intuitive estimate of both the shape and the duration of a single-particle wave packet we look at the current pulse emitted by the cavity. The sudden change of a potential, $e U(t)=\Delta \theta\left(t-t_{-}\right)$, results in a transient current pulse (an expectation value), ${ }^{2,20,37}$

$$
I_{n a}(t)=\theta\left(t-t_{-}\right) \frac{e}{\tau_{D}} e^{-\left(t-t_{-}\right) / \tau_{D}},
$$

with highly asymmetric shape (we ignore a fine structure ${ }^{37,39}$ on the scale of $\tau$ ). Here $e$ is the electron charge and $\theta(t)$ the Heaviside $\theta$ function. The time $t_{-}$denotes the time at which the potential changes, leading to the emission of an electron and the label " $n a$ " stands for nonadiabatic. Indeed, as explained below, such an emission process corresponds to nonadiabatic emission conditions. The time $\tau_{D}$,

$$
\tau_{D}=\frac{\tau}{T}
$$

the dwell time of an electron in the cavity. Therefore the dwell time sets the relevant time scale of the problem under consideration. First, the period $\mathcal{T}$ of the gate voltage, $U(t)=$ $U(t+\mathcal{T})$, should be long enough for the driven cavity to work as a single-particle source, ${ }^{2}$

$$
\mathcal{T} \gg \tau_{D}
$$

Note that to operate the source periodically, the energy level needs to be returned back to its initial position by applying the opposite potential $-\Delta \theta\left(t-t_{+}\right)$. Here, $t_{+}$denotes the time at which the emission of a hole starts. The delay between subsequent potential steps should be longer than the duration of a current pulse, $t_{+}-t_{-} \gg \tau_{D}$, to allow an electron emission to be completed: the emitted charge $q=\int_{t_{-}}^{t_{+}} I(t) d t$ should be equal to an electron charge, $q=e$. At time $t_{+}$a hole can be emitted.

Second, the dwell time $\tau_{D}$ defines the condition of adiabatic or nonadiabatic emission. If the potential $U(t)$ changes fast on the scale of $\tau_{D}$, then we speak about a nonadiabatic emission. In this case the shape of an emitted current pulse is asymmetric and given by Eq. (1). In contrast, if $U(t)$ changes smoothly compared to $\tau_{D}$, the current pulse is predicted to be symmetric. ${ }^{18,20}$ Close to $t_{-}$, the corresponding current pulse $I_{a d}$ reads

$$
I_{a d}(t)=\frac{e \Gamma_{\tau} / \pi}{\left(t-t_{-}\right)^{2}+\Gamma_{\tau}^{2}} .
$$

Now the duration $2 \Gamma_{\tau}$ of a current pulse is defined by the time of crossing,

$$
\Gamma_{\tau}=\frac{\delta}{|e d U / d t|_{t=t_{-}} \mid},
$$

where $2 \delta$ is the width of a quantum level in the weakly coupled cavity. In the model used $\delta=T \Delta /(4 \pi)$. For $e U(t)=$ $(\Delta / 2) \cos (\Omega t)$, where $\Omega=2 \pi / \mathcal{T}$, and $t_{-}=3 \mathcal{T} / 4$ we find

$$
\Gamma_{\tau}=\mathcal{T} \frac{T}{4 \pi^{2}} .
$$

Remarkably, it was shown in Ref. 40 that the pulse duration $2 \Gamma_{\tau}$ also sets the single-particle coherence time of an electron emitted adiabatically. This shows that the source, described by this analytical model, has no intrinsic dephasing processes. ${ }^{10,41}$ This makes the emitted single electron states of particular interest for further applications in quantum information processing.

Equation (4) is calculated assuming that ${ }^{27}$

$$
\Gamma_{\tau} \gg \tau_{D} .
$$

It means that the level of the cavity crosses the Fermi sea level so slowly that an electron has enough time to leave the cavity once his energy becomes larger than the Fermi energy.

From Eq. (7) it also follows that the width of a wave packet emitted adiabatically is much larger than the width of a wave packet emitted nonadiabatically. Apparently with decreasing crossing time $\Gamma_{\tau}$, keeping the period $\mathcal{T}$ large compared to $\tau_{D}$, the shape of the pulse evolves from adiabatic, Eq. (4), to nonadiabatic, Eq. (1). For a level driven with a constant speed, an analysis describing this crossover can be found in Ref. 20.

The current pulses $I_{n a}(t)$, Eq. (1), and $I_{a d}(t)$, Eq. (4), have both similar and different features. On one hand, they both carry a quantized charge. Therefore, we anticipate that they both should show similar quantization effects ${ }^{18-21}$ and effects arising due to the overlap of wave packets. ${ }^{18,22-25,29}$ We use below the shot-noise suppression effect as an example.

On the other hand, for some effects the shape of a wave packet is crucial. As an example below we use the effect of reabsorption ${ }^{27,28}$ predicted for the adiabatic regime: If two cavities are coupled to the same edge state, then the electron emitted adiabatically by one cavity can be reabsorbed by another cavity emitting a hole at the same time; see Fig. 2(a). First of all, in this regime the time-dependent current is zero, $I(t)=0 .{ }^{27}$ This current consists of two parts, electron, $I^{e}(t)$, and hole, $I^{h}(t)$, which compensate each other: $I^{e}(t)=-I^{h}(t) \rightarrow I(t)=I^{e}(t)+I^{h}(t)=0$. To clarify whether it is merely a compensation effect or a reabsorption effect, additionally the heat generated by the two cavities was analyzed. ${ }^{28}$ It was shown that each particle, either an electron or a hole, carries an excess energy (over the Fermi energy)

$$
\mathcal{E}_{a d}=\frac{\hbar}{2 \Gamma_{\tau}} .
$$

This energy can be understood as the work done by the potential $U(t)$ on the particle during its escape from the cavity. The particle starts to escape when its energy becomes equal to the Fermi energy. The time it takes to escape is the dwell 


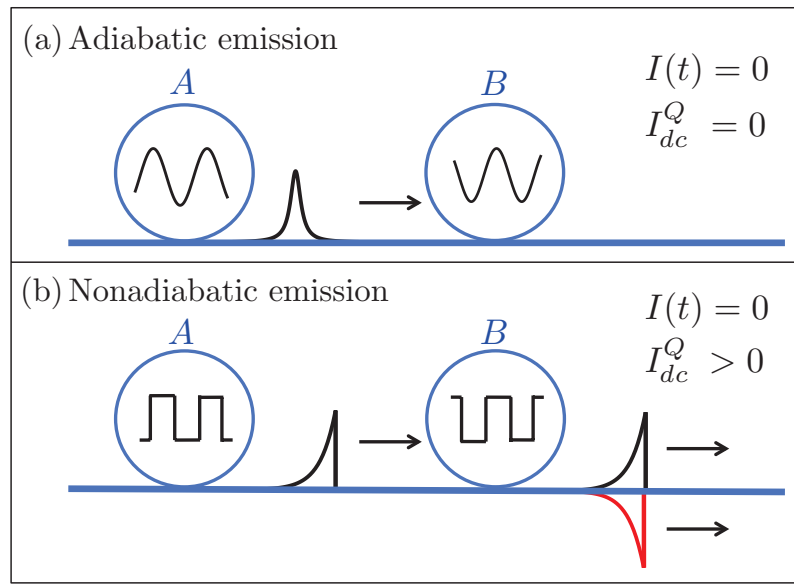

FIG. 2. (Color online) The two-particle emitter consists of two cavities specified by same parameters and coupled to the same chiral edge state. Electrons propagate along edge states shown as blue solid lines. Cavity $B$ is tuned to emit a hole at the time when the electron emitted by cavity $A$ reaches cavity $B$. (a) Adiabatic emission: when the driven potential is slow and smooth, an electron emitted by the cavity A is completely reabsorbed by cavity $B$. The re-absorption process is the time-reversed emission process which is possible because of the symmetric shape of the single-particle states. Both the charge current $I(t)$ and the dc heat flow $I_{d c}^{Q}$ nullify. (b) Nonadiabatic emission: the cavities are driven by pulsed potentials and electron-hole pairs are emitted. Since this pair is neutral, the time-dependent current is zero, $I(t)=0$. However, both the electron and the hole carry energy. Because of the asymmetric shape of the pulses reabsorption cannot be a time-reversed emission process. There is no absorption effect, the generated dc heat flow is not zero: $I_{d c}^{Q}>0$.

time, $\tau_{D}=h /(T \Delta)$, given in Eq. (2). We use Eq. (5) and find $\mathcal{E}_{a d}=\tau_{D}|e d U / d t|_{t=t_{-}} \mid$. Notice the energy of a particle in the cavity has an uncertainty $\delta$ (the level width). This results in the uncertainty $\Gamma_{\tau}$ of the time when a particle starts to escape the cavity. That in turns defines the width of the current pulse, Eq. (4).

If two cavities emit an electron and a hole at different times, then these two particles together carry the energy $2 \mathcal{E}_{a d}$. However, if an electron and a hole are emitted at the same time (the time of flight between the cavities should be trivially taken into account) then the extra energy flowing out of the system is zero. ${ }^{28}$ Clearly this means that an electron emitted by the cavity $A$ and carrying an energy $\mathcal{E}_{a d}$ was reabsorbed by the cavity $B$. This effect is paradoxical: On one hand, in fact, the hole emission is an electron absorption. On the other hand, the cavity $B$ can absorb any electrons in the waveguide passing it. Why does it absorb the electron emitted by the cavity $A$ ?

Possibly this effect can be understood using time-reversal symmetry arguments. First, let us take only one cavity and let it emit an electron. After that let us reverse time. Apparently the emitted electron will be reabsorbed. Importantly, the portion of the wave packet emitted last will be reabsorbed first. Now let us take two identical cavities and let us drive them with potentials $U_{1}(t)$ and $U_{2}(t)$ related by the time-reversal symmetry, $U_{2}(t)=U_{1}(-t)$. Note with such potentials if the first cavity emits an electron the second cavity emits a hole and vice versa. We can expect the second cavity to be an analog of the time-reversal twin of the first cavity. To make such an analogy complete, the shape of the wave packet does matter, because the second cavity will first reabsorb (if possible) the part of the wave packet which was emitted first. In contrast, the true time-reversal twin will first absorb what was emitted last. If the shape of a wave packet is symmetric, as in the adiabatic emission regime [for the corresponding current pulse see Eq. (4)], then there is no difference between what was emitted first and what was emitted last. Consequently the second cavity can play the role of the time-reversal twin and reabsorb what was emitted by the first cavity.

However, if the shape of a wave packet is nonsymmetric, as in the nonadiabatic emission regime [for the corresponding current pulse see Eq. (1)], then there is a striking difference between what was emitted first and last. As a consequence what the second cavity sees is different from what the time-reversal twin would see. Therefore, adiabatic and nonadiabatic cavities work differently. As we show below, in the nonadiabatic emission regime both cavities emit together an electron-hole pair, which carries no charge, $I(t)=0$, but carries a nonzero energy.

The paper is organized as follows: In Sec. II we discuss the shot-noise quantization and the shot-noise suppression effect for electrons emitted nonadiabatically. In Sec. III the $\mathrm{dc}$ heat flow generated by the two-particle emitter working in the nonadiabatic regime is analyzed. We conclude with a brief discussion in Sec. IV. Details of the calculations are in the appendixes. In Appendix A we derive the Floquet scattering amplitude for a cavity driven by the pulsed potential. In Appendix A the zero-frequency correlation function for currents flowing through the electron collider circuit is calculated. In Appendix A we discuss the dc heat flow generated by the two-cavity emitter.

\section{PAULI SUPPRESSION OF SHOT NOISE}

A mesoscopic electron collider is a circuit in which electrons incident from different leads can meet and collide ${ }^{42-45}$ at a wave splitter (a mesoscopic quantum point contact). We consider a collider with two single-electron emitters, $A$ and $B$, weakly coupled to the two chiral edge states, the electron waveguides, which are in turn coupled via a quantum point contact $C$ (QPC $C$ ) with transmission probability $T_{C}$, Fig. 3 . Each cavity $j=A, B$ is driven by the periodic potential, $U^{j}(t)=U^{j}(t+\mathcal{T})$, with the same period $\mathcal{T}=2 \pi / \Omega$. For simplicity we use the cavities with identical parameters but emitting particles possibly at different times. Four metallic contacts, 1-4, playing the role of electron reservoirs are kept at the same electrochemical potential $\mu$ and zero temperature.

We assume that the periodic potential, $U^{j}(t)=U^{j}(t+\mathcal{T})$, of the two sources, $j=A, B$, changes between two constant values in a steplike manner,

$$
U^{j}(t)= \begin{cases}U_{0}, & -\mathcal{T} / 2<t<t_{-}^{j}, \\ U_{0}+\Delta / e, & t_{-}^{j}<t<t_{+}^{j}, \\ U_{0}, & t_{+}^{j}<t<\mathcal{T} / 2 .\end{cases}
$$

The minimal value $U_{0}$ is chosen such that the Fermi level lies exactly in the middle between the two quantum levels of the 


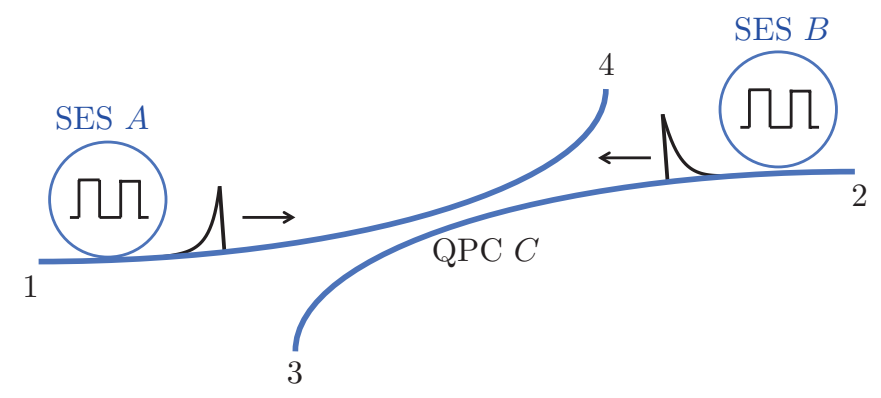

FIG. 3. (Color online) A mesoscopic electron collider. Two single-electron sources, $A$ and $B$, driven by pulsed potentials emit electrons into the waveguides. The emitted electrons collide at quantum point contact $C$. The metallic reservoirs are labeled by number from 1 to 4 .

cavity. We recall that $\Delta$ is the energy-level spacing, which is assumed to be the same for each cavity.

\section{A. Current correlation function}

We are interested in the zero-frequency correlation function $\mathcal{P}_{34}$ for currents outgoing to reservoirs (contacts) 3 and 4 . This correlation function reads 44

$\mathcal{P}_{34}=\int_{0}^{\mathcal{T}} \frac{d t}{\mathcal{T}} \int_{-\infty}^{\infty} d t^{\prime} \frac{\left\langle\delta \hat{I}_{3}(t) \delta \hat{I}_{4}\left(t+t^{\prime}\right)+\delta \hat{I}_{4}\left(t+t^{\prime}\right) \delta \hat{I}_{3}(t)\right\rangle}{2}$,

where $\delta \hat{I}_{\beta}(t)=\hat{I}_{\beta}(t)-I_{\beta}(t)$ is an operator of fluctuations of a current entering the contact $\beta=3,4, I_{\beta}(t)=\left\langle\hat{I}_{\beta}(t)\right\rangle$ is a time-dependent current flowing to the contact $\beta$. The angle brackets $\langle\cdots\rangle$ stand for quantum statistical averaging over the equilibrium state of electrons incoming from contacts $\alpha=$ 1,2 . We stress that all the contacts from 1 to 4 are disconnected from each other. Therefore, the correlators between incoming currents from contacts $\alpha=1,2$ and outgoing currents to contacts $\beta=3,4$ are zero and they do not enter Eq. (10).

The current operator $\hat{I}_{\beta}(t)$ is expressed in terms of creation/annihilation operators, $\hat{b}_{\beta}^{\dagger}(E) / \hat{b}_{\beta}(E)$, for particles outgoing to contact $\beta:^{42}$

$$
\hat{I}_{\beta}=\frac{e}{h} \int d E d E^{\prime} e^{i\left[\left(E-E^{\prime}\right) / \hbar\right] t}\left\{\hat{b}_{\beta}^{\dagger}(E) \hat{b}_{\beta}\left(E^{\prime}\right)-a_{\beta}^{\dagger}(E) \hat{a}_{\beta}\left(E^{\prime}\right)\right\} .
$$

The operator $\hat{b}$ is related to operators $\hat{a}_{\alpha}$ for particles incoming from reservoirs $\alpha=1,2$ via the Floquet scattering matrix ${ }^{46}$ of the circuit, $\hat{S}_{F}^{\text {cir }}$,

$$
\hat{b}_{\beta}(E)=\sum_{\alpha=1}^{2} \sum_{n=-\infty}^{\infty} S_{F, \beta \alpha}^{\mathrm{cir}}\left(E, E_{n}\right) \hat{a}_{\alpha}\left(E_{n}\right),
$$

where $E_{n}=E+n \hbar \Omega, n$ is an integer. The Floquet scattering matrix element $S_{F, \beta \alpha}^{\mathrm{cir}}\left(E, E_{n}\right)$ is a quantum-mechanical amplitude (properly normalized ${ }^{1}$ ) for an electron to enter the circuit from the contact $\alpha$ with energy $E_{n}$ and to leave the circuit through to the contact $\beta$ with energy $E$. Since our circuit is driven by the time-dependent potentials, an electron can change its energy during propagation through the circuit. Since the reservoirs are in equilibrium the fermionic operators
$\hat{a}_{\alpha}(E)$, first, obey the following anticommutation relation:

$$
\left[\hat{a}_{\alpha}^{\dagger}(E), \hat{a}_{\gamma}\left(E^{\prime}\right)\right]=\delta_{\alpha, \gamma} \delta\left(E-E^{\prime}\right)
$$

where $\delta_{\alpha, \gamma}$ is the Kronecker symbol while $\delta\left(E-E^{\prime}\right)$ is the Dirac $\delta$ function. Second, the average of the product of creation and annihilation operators is given by the Fermi distribution function $f(E)$, which is assumed to be the same for all the contacts,

$$
\left\langle\hat{a}_{\alpha}^{\dagger}(E) \hat{a}_{\gamma}\left(E^{\prime}\right)\right\rangle=\delta_{\alpha, \gamma} \delta\left(E-E^{\prime}\right) f(E) .
$$

Using Eqs. (11)-(14) we express the correlation function, Eq. (10), in terms of the Floquet scattering matrix elements,

$$
\begin{aligned}
\mathcal{P}_{34}= & \frac{e^{2}}{h} \int_{0}^{\infty} d E \sum_{\gamma=1}^{2} \sum_{\delta=1}^{2} \sum_{n, m=-\infty}^{\infty} \frac{\left[f\left(E_{n}\right)-f\left(E_{m}\right)\right]^{2}}{2} \\
& \times \sum_{p=-\infty}^{\infty} S_{F, 3 \gamma}^{\mathrm{cir} *}\left(E, E_{n}\right) S_{F, 3 \delta}^{\mathrm{cir}}\left(E, E_{m}\right) \\
& \times S_{F, 4 \delta}^{\mathrm{cir} *}\left(E_{p}, E_{m}\right) S_{F, 4 \gamma}^{\mathrm{cir}}\left(E_{p}, E_{n}\right) .
\end{aligned}
$$

The Floquet scattering matrix of the circuit, $\hat{S}_{F}^{\text {cir }}$, is expressed in terms of the Floquet scattering matrices, $\hat{S}_{F}^{j}$ of the sources $j=A$ and $j=B$. The element $S_{F}^{j}\left(E_{n}, E\right)$ is a forward scattering amplitude for electrons in the chiral waveguide to pass through the place where the source $j$ is coupled to and to change their energy from $E$ to $E_{n}=E+n \hbar \Omega$. Here $\Omega$ is the frequency of the potential driving the source $j$. Therefore, $\hat{S}_{F}^{j}$ is a scalar in an orbital space and a matrix in an energy space. In contrast, the Floquet scattering matrix of the entire circuit, $\hat{S}_{F}$, is a matrix in both the orbital and energy spaces. The elements of $\hat{S}_{F}$ and $\hat{S}_{F}^{j}$ are related as follows:

$$
\begin{aligned}
& S_{F, 31}^{\mathrm{cir}}\left(E_{p}, E_{n}\right)=t_{C} e^{i \varphi_{L^{A}}(E)} e^{i p \Omega \tau_{L^{A}}} S_{F}^{A}\left(E_{p}, E_{n}\right), \\
& S_{F, 32}^{\mathrm{cir}}\left(E_{p}, E_{n}\right)=r_{C} e^{i \varphi_{L^{B}}(E)} e^{i p \Omega \tau_{L^{B}}} S_{F}^{B}\left(E_{p}, E_{n}\right), \\
& S_{F, 41}^{\mathrm{cir}}\left(E_{p}, E_{n}\right)=r_{C} e^{i \varphi_{L^{A}}(E)} e^{i p \Omega \tau_{L^{A}}} S_{F}^{A}\left(E_{p}, E_{n}\right), \\
& S_{F, 42}^{\mathrm{cir}}\left(E_{p}, E_{n}\right)=t_{C} e^{i \varphi_{L^{B}}(E)} e^{i p \Omega \tau_{L^{B}}} S_{F}^{B}\left(E_{p}, E_{n}\right),
\end{aligned}
$$

where $r_{C} / t_{C}$ is the reflection/transmission amplitude at QPC $C$ assumed to be energy independent, $L^{j}$ is the distance to the cavity $j=A, B$ from QPC $C, \varphi_{L^{j}}(E)$ is the phase factor corresponding to free propagation from the cavity $j$ to QPC $C, \tau_{L^{j}}$ is the time of flight from the cavity $j$ to QPC $C$. We assume linear dispersion for free electrons and, therefore, use $\varphi_{L^{j}}\left(E_{p}\right)=\varphi_{L^{j}}(E)+p \Omega \tau_{L^{j}}$. We dropped unimportant phase factors related to free propagation from the metallic contact 1 or 2 to QPC $C$ and from QPC $C$ to the contacts 3 or 4 . Other elements of $\hat{S}_{F}$ are zero due to the chirality of the electron motion.

The Floquet scattering matrix elements $\hat{S}_{F}^{j}\left(E_{n}, E\right)$ of the source driven by the step potential $U^{j}(t)$, Eq. (9), is given in Eq. (A20) where for the sake of short notation the upper index $j$ is omitted. Substituting that equation into Eqs. (16) and then into Eq. (15) we calculate the current correlation function $\mathcal{P}_{34}$. The detail of calculations are presented in Appendix A. Here we discuss the results. 


\section{B. Quantized noise of a single source}

Let us for a moment switch off one of the sources. Then we find [see Eq. (B5)]

$$
\mathcal{P}_{34}^{n a}=-2 \mathcal{P}_{0},
$$

where $\mathcal{P}_{0}=e^{2} T_{C}\left(1-T_{C}\right) / \mathcal{T}$ and the superscript " $n a$ " indicates a nonadiabatic regime. We stress that $T_{C}$ is the transmission probability of QPC $C$ connecting the two waveguides as shown in Fig. 3. The result, Eq. (17), coincides completely with the one found for the cavity emitting particles adiabatically ${ }^{18}$ and, therefore, it tells us that at zero temperature the zerofrequency current correlation function is independent of the parameters of both the cavity and driving potential as far as the cavity emits separate particles. The quantity $\mathcal{P}_{34}^{n a}$, Eq. (17), at zero temperature can be interpreted as due to the shot noise of two indivisible quanta, one electron and one hole, emitted during each period and scattered at quantum point contact $C$ to either the contact 3 or 4 . Such a partition noise was measured in Ref. 6. The deviation from the theoretical prediction found is attributed to the effect of a nonzero temperature.

If the source emits $N$ electron and $N$ holes during the period then the factor 2 in Eq. (17) is replaced by the factor $2 N$. We also note that the noise per particle, $-\mathcal{P}_{0}$, is just the result of the partition noise of a dc source biased with the voltage $e V=\hbar \Omega$; see, e.g., Ref. 44. Let us now consider the situation where the sources $A$ and $B$ are both operating as shown in Fig. 3.

\section{Shot-noise suppression effect}

If both sources work then the total shot noise depends crucially on whether or not two electrons (respectively two holes) emitted by the different sources pass QPC $C$ at different times. If the particles pass quantum point contact $C$ at different times, $\left|t_{\mp}^{A}-t_{\mp}^{B}\right| \gg \tau_{D}$, then the shot noise is $\mathcal{P}_{34}^{n a}=-4 \mathcal{P}_{0}$, since both sources together emit four particles, two electrons and two holes, during each period. Due to the Pauli principle the noise is reduced when particles arrive nearly simultaneously at the QPC. This leads to the Pauli peak for the cross correlator, see Fig. 4, or the Pauli dip in the autocorrelator. The Pauli peak is the fermionic analog of the bosonic Hong-Ou-Mandel ${ }^{47}$ dip known in optics. We describe the aforementioned reduction with a function $D(\delta t)$ dependent on the difference of arrival times $\delta t=t_{\mp}^{A}-t_{\mp}^{B}$. The calculations presented in Appendix B 2 give for the nonadiabatic case

$$
\begin{aligned}
\frac{\mathcal{P}_{34}^{n a}}{\mathcal{P}_{0}} & =-2 D^{n a}\left(t_{-}^{A}-t_{-}^{B}\right)-2 D^{n a}\left(t_{+}^{A}-t_{+}^{B}\right), \\
D^{n a}(\delta t) & =1-e^{-|\delta t| / \tau_{D}},
\end{aligned}
$$

where we chose the sources to be placed the same distance from the QPC $C$. Remember we assumed that the two cavities emit wave packets of the same shape. For the case of cavities emitting nonadiabatically wave packets of different shape, see Ref. 48.

The behavior of the shot noise discussed above qualitatively agrees with what we predicted for emitters working under

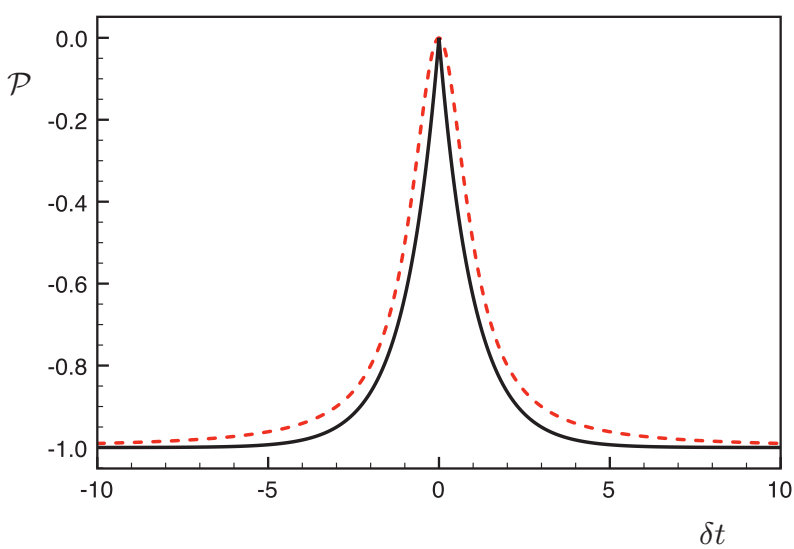

FIG. 4. (Color online) The Pauli peak: The shot noise per particle of an electron collider, $\mathcal{P}=\mathcal{P}_{34} /\left(2 N \mathcal{P}_{0}\right)$, as a function of the time delay $\delta t \equiv t_{-}^{A}-t_{-}^{B}=t_{+}^{A}-t_{+}^{B}$ normalized by $\Gamma_{\tau}$ for an adiabatic emission, Eq. (19) (red dashed line), and by $\tau_{D}$ for a nonadiabatic emission, Eq. (18) (black solid line).

adiabatic conditions: ${ }^{18}$

$$
\begin{aligned}
\frac{\mathcal{P}_{34}^{a d}}{\mathcal{P}_{0}} & =-2 D^{a d}\left(t_{-}^{A}-t_{-}^{B}\right)-2 D^{a d}\left(t_{+}^{A}-t_{+}^{B}\right), \\
D^{a d}(\delta t) & =1-\frac{4 \Gamma_{\tau}^{2}}{(\delta t)^{2}+4 \Gamma_{\tau}^{2}} .
\end{aligned}
$$

Here the superscript "ad" indicates adiabatic emission conditions.

The reduction function $D(\delta t)$ can also be calculated from the overlap of wave functions of colliding particles at the quantum point contact (the wave splitter). ${ }^{44,49}$ If the two sources emit wave packets of the same shape then the overlap can be formally expressed in terms of the single-particle correlation function $G_{e}^{(1)}\left(t_{1}, t_{2}\right)$ discussed in Ref. 10. The reduction function then can be written as follows:

$$
D(\delta t)=1-v_{\mu}^{2}\left|\int d t G_{e}^{(1)}(t+\delta t, t)\right|^{2},
$$

where the integral runs over the time interval when the particles pass the QPC and $v_{\mu}$ is a velocity of an electron in the waveguide evaluated at the Fermi energy $\mu$. The factor $v_{\mu}^{2}$ is introduced to account for the wave-function normalization in such a way that at the complete overlap, $\delta t=0$, the reduction function $D=0$.

For the definition of the correlation function for electrons emitted by the source coupled to the chiral waveguide, see Fig. 1, we refer to Ref. 10 by the same authors: $G_{e}^{(1)}\left(t_{1}, t_{2}\right)=$ $\left\langle\hat{\Psi}^{\dagger}\left(t_{1}\right) \hat{\Psi}\left(t_{2}\right)\right\rangle-\left\langle\hat{\Psi}^{\dagger}\left(t_{1}\right) \hat{\Psi}\left(t_{2}\right)\right\rangle_{0}$, where the subscript 0 indicates that the single-electron source is switched off. For our purposes the field operators $\hat{\Psi}, \hat{\Psi}^{\dagger}$ for electrons in the waveguide are calculated at different times, $t_{1}, t_{2}$, but at the same spatial coordinates somewhere after the source. Expressing the field operators in terms of operators $\hat{b}$ for electrons scattered off the cavity (if the potential driving the cavity is switched on) or in terms of operators $\hat{a}$ describing electrons incoming from the reservoir (if the potential driving 
the cavity is switched off), ${ }^{42}$ we find

$$
\begin{aligned}
G_{e}^{(1)}\left(t_{1}, t_{2}\right)= & \iint d E_{1} d E_{2} \frac{e^{i\left(E_{1} t_{1}-E_{2} t_{2}\right) / \hbar}}{\sqrt{v\left(E_{1}\right) v\left(E_{2}\right)}} \\
& \times\left\langle\hat{b}^{\dagger}\left(E_{1}\right) \hat{b}\left(E_{2}\right)-\hat{a}^{\dagger}\left(E_{1}\right) \hat{a}\left(E_{2}\right)\right\rangle,
\end{aligned}
$$

where $v(E)$ is the density of states of electrons in the waveguide. For a circuit consisting of a single periodically driven source coupled to a single waveguide, see Fig. 1, the $\hat{b}$ operators are related to $\hat{a}$ operators as follows:

$$
\hat{b}(E)=\sum_{n=-\infty}^{\infty} S_{F}\left(E, E_{n}\right) \hat{a}\left(E_{n}\right)
$$

where $S_{F}\left(E, E_{n}\right)$ is the Floquet scattering matrix of the source. In the adiabatic regime the Floquet scattering matrix $S_{F}\left(E_{n, E}\right)=S_{n}(E)$ is defined by the Fourier transform $S_{n}(E)$ of the frozen scattering amplitude, Eq. (A5a). Using it in Eq. (22) and then in Eq. (21), we obtain in the zero-temperature limit

$$
G_{e, a d}^{(1)}(t+\delta t, t)=\frac{1}{\pi \Gamma_{\tau} v_{\mu}} \frac{1}{\left(1-i \frac{t+\delta t}{\Gamma_{\tau}}\right)\left(1+i \frac{t}{\Gamma_{\tau}}\right)} .
$$

For the nonadiabatic emission we use Eq. (A20) and find

$$
G_{e, n a}^{(1)}(t+\delta t, t)=e^{-i(\Delta / 2)(\delta t / \hbar)} \theta(t) \theta(\delta t) \frac{e^{-(t+\delta t / 2) / \tau_{D}}}{\tau_{D} v_{\mu}} .
$$

By inserting Eqs. (23) and (24) into Eq. (20), we recover respectively the reduction factor in the adiabatic regime, Eq. (19b), and in the nonadiabatic regime, Eq. (18b).

In Fig. 4 we show $\mathcal{P}_{34}^{n a}$ and $\mathcal{P}_{34}^{a d}$ as functions of the time difference $\delta t \equiv \delta t_{-}=\delta t_{+}\left(\right.$with $\left.\delta t_{\chi}=t_{\chi}^{A}-t_{\chi}^{B}, \chi=\mp\right)$ normalized on $\tau_{D}$ and $\Gamma_{\tau}$, respectively. The Pauli peaks are remarkably similar under the two limiting operating conditions despite the fact that the emitted states are very different. However the two operating conditions can be perhaps differentiated experimentally taking a closer look at the top of a peak. It is sharp in the nonadiabatic case and smoother in the adiabatic case. We remark that for different incident states the Pauli peak has an asymmetric shape in the nonadiabatic case $\mathrm{c}^{48}$ but remains symmetric in the adiabatic case. ${ }^{18}$ Measurements on an electronic collider have now succeeded in detecting the Pauli peak. ${ }^{50}$

\section{TWO-PARTICLE EMITTER}

In this section we consider the circuit with two cavities connected in series to the same edge state as shown in Fig. 2(b). If both cavities emit particles at close times, such a circuit serves as a two-particle emitter. Its work under the adiabatic condition of emission was analyzed in Refs. 27 and 28. Here we analyze it under the nonadiabatic condition of emission when each cavity is driven by the pulsed potential; see Eq. (9).

We calculate the dc heat flow $I_{d c}^{Q}$ generated by the two-cavity source as a quantity able to differentiate various cases: (i) separate emission of particles, (ii) electron-hole emission, (iii) two-electron (two-hole) emission. The details of calculations are given in Appendix C. Here we discuss the results.
We start from conditions when the cavities emit particles at different times; see Appendix C 2a. The dc heat flow, $I_{d c}^{Q}=$ $4 \mathcal{E}_{\text {na }} / \mathcal{T}$, is due to four particles emitted by both cavities during each period. Each particle carries an excess (over the Fermi sea level) energy

$$
\mathcal{E}_{n a}=\frac{\Delta}{2} .
$$

The above result is clear, since the potential $U^{j}(t)$, Eq. (9), moves a quantum level of the cavity $j$ by $\Delta / 2$ above (below) the Fermi energy when an electron (a hole) is emitted.

The single-particle energy $\mathcal{E}$ can be also understood on the base of the Joule-Lenz law,

$$
\mathcal{E}=R \int d t I^{2}(t)
$$

Here we integrate over a single-particle current pulse. Under the adiabatic emission condition we use Eq. (4) for $I(t)$ and Eq. (8) for $\mathcal{E}$ and find from Eq. (26) that the relevant resistance, $R_{a d} \equiv R_{q}=h /\left(2 e^{2}\right)$, is the charge relaxation resistance quantum ${ }^{38,51-54}$ which appears in the linear response (admittance) of the cavity ${ }^{34}$ (a quantum capacitor) at lowtemperature. Under the nonadiabatic emission condition we use Eq. (1) for $I(t)$ and Eq. (25) for $\mathcal{E}$. From Eq. (26) we then find $R_{n a}=h /\left(e^{2} T\right)$. This is an ordinary two-terminal resistance of the (single channel) quantum point contact connecting the cavity to the electron waveguide. This resistance was found experimentally in the optimal operation conditions ${ }^{2}$ and it appears in theory in both the high-temperature ${ }^{37}$ and incoherent ${ }^{55}$ case. Therefore, we see that the factor $R$ in Eq. (26) is not universal but it depends crucially on the way an electron is emitted.

Note also that according to Ref. 56 the adiabatic source is optimal in the sense that it dissipates the minimal heat per generated particle (an electron or a hole), $R_{a d}=R_{q}$. In contrast the nonadiabatic source dissipates more energy, $R_{n a} \gg R_{q}$ and thus it is nonoptimal. It is worthwhile to mention that the criteria for an optimal pump generating a dc quantized current $^{56}$ works also in our case for the emitter which produces a quantized ac current, a sequence of alternating electrons and holes.

Now we come back to the two-particle emitter. If two cavities emit two electrons (two holes) simultaneously, see Appendix C 2c, the energy carried by the pair of particles, $\mathcal{E}_{n a}^{e e}=\mathcal{E}_{n a}^{h h}$, is enhanced two times compared to the condition of independent emission (when two separate particles carry energy $2 \mathcal{E}_{n a}$ ):

$$
\mathcal{E}_{n a}^{e e}=4 \mathcal{E}_{n a} .
$$

The same two-time enhancement was found under the adiabatic emission condition. ${ }^{28}$ The enhancement factor 2 can be understood using the Joule-Lenz law, Eq. (26), since if the two particles are emitted simultaneously, then the amplitude of a current pulse is doubled. The reason that an energy enhancement cannot be avoided follows from the Pauli blocking: Cavity $B$ cannot emit a particle with energy $\mathcal{E}$ if there is a particle with the same energy (emitted by cavity $A$ ). Therefore, cavity $B$ has to emit a particle with an enhanced energy. Under the nonadiabatic condition cavity $B$ excites an electron to the next available quantum level of the cavity 
and then an electron having energy $\Delta / 2+\Delta$ leaves a cavity. This scenario agrees with a nonadiabatic excitation of an electron in a dynamical quantum dot observed in Ref. 57. The direct spectroscopy of energies of electrons emitted by the two-particle source can be done in the same way as proposed in Ref. 58 for the single-particle emitter.

The last operating condition we want to discuss is, see Appendix $\mathrm{C} 2 \mathrm{~b}$, when one cavity emits an electron at the time the other one emits a hole; see Fig. 2(b). We find that the dc heat flow is not changed compared to the case when the particles are emitted at different times. This means that now our source emits electron-hole pairs each carrying finite heat $\mathcal{E}_{n a}^{e h}=2 \mathcal{E}_{n a}$ but zero charge. This is in striking contrast with the adiabatic emission case when a particle emitted by one source is reabsorbed by the other source thus nullifying both the charge current and the dc heat flow. ${ }^{27,28}$ The nullification of the dc heat flow can also be understood as a work transfer between the external forces ${ }^{59,60}$ driving the two particle sources. We remark that in the electron-hole emission case the Joule-Lenz law, Eq. (26), holds under the adiabatic condition, whereas it seems to be violated under the nonadiabatic condition. Note that also the fluctuation-dissipation relation is broken in the adiabatic operating conditions when the two cavities generate electron-hole pairs. ${ }^{28}$

\section{CONCLUSION}

Here we developed an analytical Floquet scattering matrix approach to describe the chiral single-electron source driven by the pulsed potential and, therefore, emitting particles, electrons and holes, nonadiabatically. We analyzed an electronic collider and the two-particle emitter circuits with such sources and compared them to the analogous circuits with single-electron emitters driven by the smooth potential, i.e., emitting particles adiabatically.

We found that the collision of electrons approaching a quantum point contact from different sides, see Fig. 3, suppresses the shot noise. This effect is similar to the one found under the adiabatic emission condition ${ }^{18}$ and it is due to the Pauli repulsion between the overlapping electrons, which forces them to go to the different outputs thus regularizing the outgoing particle flows. The sharper suppressing factor, Fig. 4 (black solid line), is due to the spatial asymmetry of traveling wave packets generated nonadiabatically.

A more striking difference was found for a circuit comprising two cavities connected to the same edge state, Fig. 2. If both cavities emit particles at close times such a circuit serves as a two-particle emitter. The difference between adiabatic and nonadiabatic emission conditions appears when cavities emit particles of a different kind, i.e., one cavity emits a hole at the same time as the other cavity emits an electron. If particles are emitted adiabatically, then cavity $B$ reabsorbs ${ }^{27,28}$ what was emitted by cavity $A$, whereas in the nonadiabatic emission mode a neutral electron-hole pair having a finite energy is emitted. This can be verified by looking at the dc heat flowing out of the system: It is zero under the adiabatic emission condition but finite under the nonadiabatic one. If both cavities emit particles of the same kind (two electrons or two holes) then under either adiabatic or nonadiabatic emission conditions we found doubling of heat compared to the case when all particles are emitted at different times.

Surprisingly the Joule-Lenz law relating a current through and heat released in the macroscopic conductor also holds for the single-particle excitation: The square of the single electron (hole) current pulse integrated over time gives the heat carried by this particle from the source and released in the macroscopic reservoir. This law works under either adiabatic or nonadiabatic emission conditions though with different relevant resistances. However, it is violated completely for the two-particle source emitting an electron-hole pair under the nonadiabatic emission condition.

\section{ACKNOWLEDGMENTS}

G.H. acknowledges the support of the Alexander von Humboldt Foundation in the framework of the Alexander von Humboldt Professorship, endowed by the Federal Ministry of Education and Research. This work was supported by the Swiss National Science Foundation and Swiss National Center for Competence in Research on Quantum Science and Technology QSIT.

\section{APPENDIX A: THE FLOQUET SCATTERING MATRIX}

In Ref. 37 the scattering amplitude $S_{\text {in }}(t, E)$ for an electron with a linear dispersion in a chiral waveguide being scattered off a one-dimensional circular edge state (a cavity) was calculated. The cavity is driven by the uniform in space and periodic in time potential, $U(t)=U(t+\mathcal{T})$. This amplitude can be presented as the sum of partial amplitudes classified by the number of turns $q$ made by an electron with energy $E$ entering the cavity before leaving it at time $t$ :

$$
S_{\mathrm{in}}(t, E)=r+\tilde{t}^{2} \sum_{q=1}^{\infty} r^{q-1} e^{i\left\{q \varphi(E)-\Phi_{\mathrm{in}, q}(t)\right\}} .
$$

Here $r / \tilde{t}$ is the reflection/transmission amplitude of the quantum point contact connecting the cavity and the chiral one-dimensional conductor (an electron waveguide), $\varphi(E)=$ $\varphi(\mu)+(\tau / \hbar)(E-\mu)$ is the kinematic phase picked up by an electron during one turn in the cavity, $\Phi_{q}$ is the phase due to the time-dependent potential acquired by an electron during $q$ turns,

$$
\Phi_{\mathrm{in}, q}(t)=\frac{e}{\hbar} \int_{t-q \tau}^{t} d t^{\prime} U\left(t^{\prime}\right),
$$

where $\tau$ is the duration of one turn. Details of the derivation can be found in Ref. 1 .

The Fourier coefficients of $S_{\text {in }}$ define the elements of the Floquet scattering matrix (in the energy space),

$$
S_{F}(E+n \hbar \Omega, E)=S_{\text {in }, n}(E) \equiv \int_{0}^{\mathcal{T}} \frac{d t}{\mathcal{T}} e^{\text {in } \Omega t} S_{\text {in }}(t, E),
$$

which are amplitudes corresponding to photon-assisted scattering with exchange of $n$ energy quanta $\hbar \Omega$ between an electron and the driving field. Here $\Omega=2 \pi / \mathcal{T}$ is the frequency of the drive. For $n>0$ the electron absorbs energy whereas for $n<0$ it emits energy. 
For some calculations the dual amplitude $S_{\text {out }}(E, t)$, which fixes the time when an electron enters the dot, ${ }^{61}$ is more natural to use. Its Fourier coefficients relate to the Floquet scattering amplitudes in the following way:

$$
S_{F}(E, E-n \hbar \Omega)=S_{\text {out }, n}(E) \equiv \int_{0}^{\mathcal{T}} \frac{d t}{\mathcal{T}} e^{\text {in } \Omega t} S_{\text {out }}(E, t) .
$$

The amplitudes $S_{\text {in }}(t, E)$ and $S_{\text {out }}(E, t)$ are generally interrelated in a simple manner. ${ }^{61}$ In particular, for the model of interest here, the amplitude $S_{\text {out }}(E, t)$ is given by Eq. (A1) with $\Phi_{\text {in, } q}(t)$ replaced by

$$
\Phi_{\text {out }, q}(t)=\frac{e}{\hbar} \int_{t}^{t+q \tau} d t^{\prime} U\left(t^{\prime}\right) .
$$

Depending on the ratio between the time of a single turn $\tau$ and a characteristic time during which the driving potential $U(t)$ changes we distinguish adiabatic and nonadiabatic operating conditions.

\section{Adiabatic emission}

If the potential $U(t)$ changes slowly, i.e., the maximum relevant frequency is much smaller than $\tau^{-1}$. Thus we can keep $U\left(t^{\prime}\right)$ constant while integrating over time in Eqs. (A2) or (A4). We arrive at the frozen ${ }^{1,56}$ scattering amplitude, $S(U(t), E) \equiv$ $S_{\text {in }}(t, E)=S_{\text {out }}(E, t)$ :

$$
S(U(t), E)=-e^{i\left[\phi(U(t), E)+\theta_{r}\right]} \frac{1-\sqrt{\mathrm{R}} \mathrm{e}^{-i \phi(U(t), E)}}{1-\sqrt{\mathrm{R}} \mathrm{e}^{i \phi(U(t), E)}},
$$

where

$$
\phi(U, E)=\theta_{r}+\varphi(\mu)+2 \pi \frac{E-\mu}{\Delta}-2 \pi \frac{e U}{\Delta} ;
$$

$\sqrt{R}$ and $\theta_{r}$ are the absolute value and the phase of the reflection amplitude, $r=\sqrt{R} \exp \left(i \theta_{r}\right) ; \Delta=h / \tau$ is the level spacing in the cavity. The phase $\varphi(\mu)$ is a kinematic phase picked up by an electron with Fermi energy, $E=\mu$, during one turn in the cavity in the absence of a potential $U$. It is defined after Eq. (A1).

It is instructive to look at the scattering amplitude $S(t, E)$ as a function of its arguments, Fig. 5. The narrow chine visualizes a quantum level in the cavity moving under the action of the potential $U(t)$. At zero temperature the scattering amplitude at the Fermi energy, $E=\mu$, is sufficient to calculate the emitted current: $I_{a}(t)=-i e /(2 \pi) S \partial S^{*} / \partial t .^{56,62,63}$ Thus the cross section on Fig. 5 at $E=0$ shows us when a quantum level crosses the Fermi energy and, hence, when the current pulses appear. Importantly, the shape of peaks of the aforementioned cross section (at $T \ll 1$ ) is similar to the shape of a current pulse (up to a normalization factor). This can be easily shown if one considers the scattering amplitude close to, say, the time of an electron emission $t_{-}$. It reads ${ }^{18}$

$$
S(t, \mu)=\left(t-t_{-}+i \Gamma_{\tau}\right) /\left(t-t_{-}-i \Gamma_{\tau}\right) .
$$

Then the current $I_{a}(t)$, Eq. (4), is expressed in terms of the real part of the scattering amplitude as follows:

$$
I_{a}(t)=\frac{e}{2 \pi \Gamma_{\tau}}[1-\operatorname{Re} S(t, \mu)]
$$

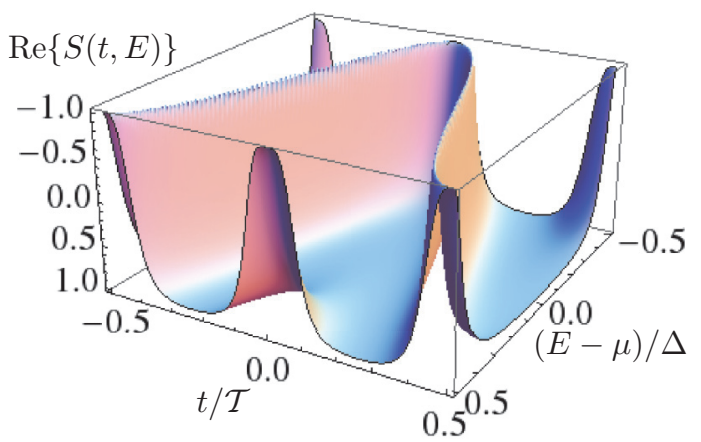

FIG. 5. (Color online) Adiabatic emission: The real part of $S(t, E)$, Eq. (A5a), is shown. The time $t$ is measured in units of the period of the drive $\mathcal{T}=2 \pi / \Omega$. The energy $E$ is measured from the Fermi energy $\mu$ in units of the level spacing $\Delta$. Only one period for both $t$ and $E$ is shown. The transmission probability of the quantum point contact connecting the cavity is $T=0.5$. Other parameters correspond to the optimal operating conditions.

\section{Nonadiabatic emission}

The periodic pulsed potential, $U(t)=U(t+\mathcal{T})$,

$$
U(t)= \begin{cases}U_{0}, & -\mathcal{T} / 2<t<t_{-}, \\ U_{1}, & t_{-}<t<t_{+}, \\ U_{0}, & t_{+}<t<\mathcal{T} / 2\end{cases}
$$

is an example, relevant to experiment, ${ }^{2}$ leading to a nonadiabatic emission. The nonadiabatic behavior is caused by the potential jumps, which formally have to be sharp on the scale of $\tau$. Before calculating the scattering amplitude for the pulsed potential $U(t)$, Eq. (A8), we consider the following auxiliary problem.

\section{a. Single-step potential}

Let us find scattering amplitudes for a cavity driven by the single-step potential,

$$
U(t)= \begin{cases}U_{0}, & t<0 \\ U_{1}, & t>0\end{cases}
$$

With this potential the time-dependent phase, say, $\Phi_{\text {in }, q}(t)$, Eq. (A2), can be easily calculated:

$$
\Phi_{\mathrm{in}, q}(t)= \begin{cases}2 \pi q \frac{e U_{1}}{h / \tau}, & t>q \tau, \\ 2 \pi \frac{e}{\tau} \frac{e \delta U}{h / \tau}+2 \pi q \frac{e U_{0}}{h / \tau}, & 0<t<q \tau, \\ 2 \pi q \frac{e U_{0}}{h / \tau}, & t<0 .\end{cases}
$$

Here $\delta U=U_{1}-U_{0}$. To sum up over $q$ in Eq. (A1) we note that for a given $t<0$ we have to use $U_{0} \forall q$. In contrast, as far as $N \tau<t<(N+1) \tau$ we have to use $U_{1}$ for $q \leqslant N$ and a more complicated phase for $q>N$. We can represent a time-dependent scattering amplitude as follows:

$$
S_{\text {in }}(t, E)=S(U(t), E)+\theta(t) \delta S_{\text {in }}(t, E) .
$$

Here $\theta(t)$ is the Heaviside $\theta$ function, $\theta(t)=0$ for $t<0$ and $\theta(t)=1$ for $t>0$. The frozen amplitude $S(U(t), E)$ is given 
by Eq. (A5), and $\delta S_{\text {in }}$ is given within each interval $N \tau<t<$ $(N+1) \tau(N=0,1,2, \ldots)$ as

$$
\begin{aligned}
\delta S_{\text {in }}(t, E)= & e^{i \theta_{r}} T R^{N / 2}\left\{\frac{e^{i(N+1) \phi\left(U_{1}, E\right)}}{1-\sqrt{\operatorname{Re}^{i \phi\left(U_{1}, E\right)}}}\right. \\
& \left.-e^{-i 2 \pi e \delta U t / h} \frac{e^{i(N+1) \phi\left(U_{0}, E\right)}}{1-\sqrt{\mathrm{R}} \mathrm{e}^{i \phi\left(U_{0}, E\right)}}\right\},
\end{aligned}
$$

with $T=1-R$ a transmission coefficient of the quantum point contact connecting the cavity to the waveguide.

The quantity $\delta S_{\text {in }}(t, E)$ characterizes how $S_{\text {in }}(t, E)$ deviates from the stationary scattering amplitude $S(U, E)$ corresponding to the instantaneous potential $U=U(t)$. This deviation exists only after the potential was changed, $t>0$, and it decreases,

$$
\delta S_{\text {in }} \sim e^{-t / 2 \tau_{D}}, \quad t \gg \tau,
$$

with a characteristic time

$$
\tau_{D}=\tau / \ln (1 / R) .
$$

An analogous calculation gives

$$
S_{\text {out }}(E, t)=S(U(t), E)+\theta(-t) \delta S_{\text {out }}(E, t) .
$$

where within each interval $-(N+1) \tau<t<-N \tau$,

$$
\begin{aligned}
\delta S_{\text {out }}(E, t)= & e^{i \theta_{r}} T R^{N / 2}\left\{\frac{e^{i(N+1) \phi\left(U_{0}, E\right)}}{1-\sqrt{\operatorname{R}} \mathrm{e}^{i \phi\left(U_{0}, E\right)}}\right. \\
& \left.-e^{-i 2 \pi e \delta U t / h} \frac{e^{i(N+1) \phi\left(U_{1}, E\right)}}{1-\sqrt{\mathrm{R}} \mathrm{e}^{i \phi\left(U_{1}, E\right)}}\right\} .
\end{aligned}
$$

In contrast to $S_{\text {in }}$ the scattering amplitude $S_{\text {out }}(E, t)$ deviates from the frozen scattering amplitude $S(E, U(t))$ at times preceding the change of a potential. At $|t| \gg \tau$ the deviation $\delta S_{\text {out }}$ decays exponentially with a characteristic time $\tau_{D}$.

\section{b. Optimal operating conditions}

The calculations are simplified greatly for the optimal operating conditions ${ }^{2,35}$ which lead to the emission of a single electron and hole during each period. One condition is that the potential changes by exactly one level spacing $\Delta=h / \tau$,

$$
e \delta U=-\chi \Delta,
$$

where $\chi=\mp$. In addition the Fermi energy should lie exactly in the middle of two neighboring quantum levels of the cavity,

$$
\theta_{r}+\varphi(\mu)-2 \pi e U_{0} / \Delta=\pi .
$$

With these conditions the frozen amplitude becomes independent of time, $S\left(U_{0}, E\right)=S\left(U_{1}, E\right)$. In other words, mere shaking of a potential would not disturb an electron system. What causes a dynamical (transient) response is electrons entering and leaving the cavity at different potentials. That is described by $\delta S_{\chi}(E, t) \equiv \delta S_{\text {in }}(t, E)=\delta S_{\text {out }}(E, t)$,

$$
\delta S_{\chi}(E, t)=e^{i \theta_{r}} T \frac{R^{N / 2} e^{i(N+1) \phi\left(U_{0}, E\right)}\left(1-e^{\chi i 2 \pi(t / \tau)}\right)}{1-\sqrt{\operatorname{R}} e^{i \phi\left(U_{0}, E\right)}},
$$

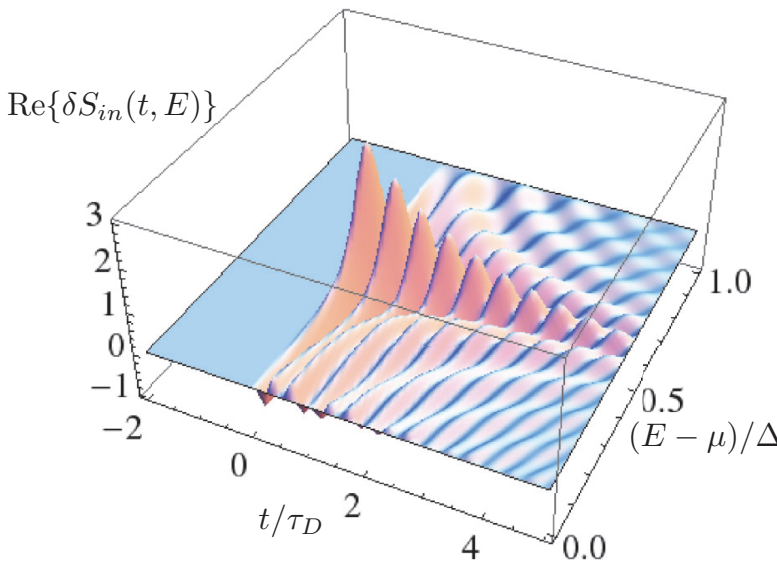

FIG. 6. (Color online) Nonadiabatic emission: The real part of $\delta S_{\text {in }}(t, E)$, Eq. (A16a), with $\chi=-$, is shown close to the time of emission of an electron, $t \sim t_{-}$. The time $t$ is measured in units of the dwell time $\tau_{D}=\tau / T$. The energy $E$ is measured from the Fermi energy $\mu$ in units of the level spacing $\Delta$. Only one period for $E$ is shown. The visible ripples reflect oscillations in time with the period of $\tau$. The transmission probability of the quantum point contact connecting the cavity is $T=0.5$. Other parameters correspond to the optimal operating conditions.

with

$$
\phi\left(U_{0}, E\right)=\pi+2 \pi \frac{E-\mu}{\Delta} .
$$

Here $\phi\left(U_{0}, E\right)=\phi\left(U_{1}, E\right)$, is a phase picked up by an electron during one turn in the cavity working under the optimal conditions. For simplicity all calculations from here on are done for optimal operating conditions. However, the formalism used in itself is not restricted to the optimal conditions, Eqs. (A15a) and (A15b).

The real part of the scattering amplitude $\delta S_{\chi}$, Eq. (A16a), is shown in Fig. 6. Its overall behavior in time reflects the asymmetry of the emitted state, in particular, of the current pulse $I_{n a}(t)$, Eq. (1). In addition it illustrates that the largest variations of the scattering amplitude occur at the energy of the quantum state in the cavity.

\section{c. Pulsed potential}

Now we come back to the periodic pulsed potential, Eq. (A8). For definiteness we use $e U_{1}>e U_{0}$. We suppose a drive with a delay between the potential steps that is long compared to the difference between absorption and emission times which in turn are taken to be long compared to the dwell time,

$$
\mathcal{T}>t_{+}-t_{-} \gg \tau_{D}
$$

Therefore the transient behavior caused by one potential step vanishes completely before the next step appears. This permits us to use the results for a single-step potential and get $(t \in$ $[-\mathcal{T} / 2, \mathcal{T} / 2])$

$$
\begin{aligned}
S_{\text {in }}(t, E)= & S\left(U_{0}, E\right)+\theta\left(t-t_{-}\right) \delta S_{-}\left(E, t-t_{-}\right) \\
& +\theta\left(t-t_{+}\right) \delta S_{+}\left(E, t-t_{+}\right), \\
S_{\text {out }}(E, t)= & S\left(U_{0}, E\right)+\theta\left(t_{-}-t\right) \delta S_{-}\left(E, t-t_{-}\right) \\
& +\theta\left(t_{+}-t\right) \delta S_{+}\left(E, t-t_{+}\right) .
\end{aligned}
$$


Note that at the time $t_{-}$an electron is emitted by the driven cavity whereas at the time $t_{+}$a hole is emitted.

\section{d. Fourier coefficients}

To calculate the Floquet scattering amplitudes, see Eqs. (A3), we need the Fourier transformation of Eq. (A18). To integrate over time we use the following trick: Since $\delta S_{\chi}$ is constant over an interval of duration $\tau$, we integrate over this interval the factor $\exp (\mathrm{in} \Omega t$ ) only and then sum up over $N$. Under the condition of Eq. (A17), the sum over $N$ runs from 0 to $\infty$. As a result we find

$$
\begin{aligned}
& S_{\mathrm{in}, n}(E)=S\left(U_{0}, E\right) \delta_{n, 0}-A_{n}(E)\left\{\frac{e^{\mathrm{in} \Omega t_{-}}}{1-\frac{n \hbar \Omega}{\Delta}}+\frac{e^{\mathrm{in} \Omega t_{+}}}{1+\frac{n \hbar \Omega}{\Delta}}\right\}, \\
& S_{\mathrm{out}, n}(E)=S_{\mathrm{in}, n}(E-n \hbar \Omega) .
\end{aligned}
$$

Here

$$
\begin{aligned}
A_{n}(E)= & S\left(U_{0}, E\right) e^{i \pi(n \hbar \Omega / \Delta)} \frac{\sin \left(\pi \frac{n \hbar \Omega}{\Delta}\right)}{\pi n} \\
& \times \frac{T}{\left(1+\sqrt{\mathrm{R}} \mathrm{e}^{i 2 \pi(E-\mu) / \Delta}\right)\left(1+\sqrt{\mathrm{R}} \mathrm{e}^{i 2 \pi(E-\mu+n \hbar \Omega) / \Delta}\right)},
\end{aligned}
$$

and $\delta_{n, 0}$ is the Kronecker symbol.

Thus substituting Eq. (A19a) into Eq. (A3a) we finally find the Floquet scattering matrix elements for the source driven by the step potential, Eq. (A8), under the optimal operating conditions, see Eqs. (A15),

$$
\begin{aligned}
S_{F}\left(E_{n}, E\right)= & S\left(U_{0}, E\right) \frac{\sin \left(\pi \frac{n \hbar \Omega}{\Delta}\right)}{\pi n} e^{i \pi(n \hbar \Omega / \Delta)} \\
& \times\left\{\delta_{n, 0}-\frac{\frac{e^{i n \Omega t_{-}}}{1-\frac{n \hbar \Omega}{\Delta}}+\frac{e^{\mathrm{in} \Omega t_{+}}}{1+\frac{n \hbar \Omega}{\Delta}}}{\rho^{*}(E) \rho\left(E_{n}\right)}\right\} .
\end{aligned}
$$

Here $E_{n}=E+n \hbar \Omega$; the scattering amplitude of the stationary cavity $S\left(U_{0}, E\right)$ is given in Eq. (A5) with $U(t)=$ $U_{0} ; \Delta$ is the level spacing in the cavity; $\rho(E)=[1+$ $\left.\sqrt{1-T} e^{i 2 \pi(E-\mu) / \Delta}\right] / \sqrt{T} ; T$ is the transparency of the quantum point contact connecting the cavity and the electron waveguide; $t_{-} / t_{+}$is the time of an electron/hole emission. We stress that under the optimal operating conditions $S_{F}(E+$ $n \hbar \Omega, E)$ is independent of both $U_{0}$ and $U_{1}$ since $S\left(U_{0}, E\right)=$ $S\left(U_{1}, E\right)$.

\section{e. Continuous frequency representation}

From Eq. (A17) it follows that $\hbar \Omega \ll \Delta$. Therefore, there are many $[n \sim \Delta /(\hbar \Omega) \gg 1]$ photon-assisted amplitudes contributing to scattering. Since the replacement $n \rightarrow n+1$ changes the scattering amplitude only a little, it is convenient to go over from the discrete frequency representation to the continuous frequency representation. For this purpose we use the following correspondence:

$$
\begin{aligned}
& n \Omega \rightarrow \Omega_{n}, \quad \sum_{n=-\infty}^{\infty} \rightarrow \int_{-\infty}^{\infty} \frac{d \Omega_{n}}{\Omega} \\
& \delta_{n, 0} \rightarrow \Omega \delta\left(\Omega_{n}\right), \quad \int_{0}^{\mathcal{T}} d t^{\prime} e^{\mathrm{in} \Omega t^{\prime}} \rightarrow \int_{-\infty}^{\infty} d t^{\prime} e^{i \Omega_{n} t^{\prime}} .
\end{aligned}
$$

Here $\delta\left(\Omega_{n}\right)$ is the Dirac $\delta$ function.
To simplify long equations we also introduce the following dimensionless quantities:

$$
\epsilon=\frac{E-\mu}{\Delta}, \quad \omega_{n}=\frac{\hbar \Omega_{n}}{\Delta},
$$

and the abbreviation

$$
\rho(\epsilon)=\frac{1+\sqrt{\operatorname{R}} e^{i 2 \pi \epsilon}}{\sqrt{T}} .
$$

With these definitions Eq. (A19a), originally expressed as a discrete Fourier transformation, now takes the form of a continuous Fourier transformation,

$$
\begin{aligned}
S_{\text {in }}\left(\omega_{n}, \epsilon\right)= & S\left(\frac{U_{0}}{\Delta}, \epsilon\right) \frac{\hbar \Omega}{\Delta} \frac{\sin \left(\pi \omega_{n}\right)}{\pi \omega_{n}} e^{i \pi \omega_{n}} \\
& \times\left\{\delta\left(\omega_{n}\right)-\frac{\frac{e^{i 2 \pi \omega_{n}\left(t_{-} / \tau\right)}}{1-\omega_{n}}+\frac{e^{i 2 \pi \omega_{n}\left(t_{+} / \tau\right)}}{1+\omega_{n}}}{\rho^{*}(\epsilon) \rho\left(\epsilon+\omega_{n}\right)}\right\}, \\
S_{\text {out }}\left(\epsilon, \omega_{n}\right)= & S\left(\frac{U_{0}}{\Delta}, \epsilon\right) \frac{\hbar \Omega}{\Delta} \frac{\sin \left(\pi \omega_{n}\right)}{\pi \omega_{n}} e^{-i \pi \omega_{n}} \\
& \times\left\{\delta\left(\omega_{n}\right)-\frac{\frac{e^{i 2 \pi \omega_{n}\left(t_{-} / \tau\right)}}{1-\omega_{n}}+\frac{e^{i 2 \pi \omega_{n}\left(t_{+} / \tau\right)}}{1+\omega_{n}}}{\rho^{*}(\epsilon) \rho\left(\epsilon-\omega_{n}\right)}\right\} .
\end{aligned}
$$

Note that here we used the following property of the Dirac $\delta$ function: $\delta\left(\omega_{n} \Delta / \hbar\right)=(\hbar / \Delta) \delta\left(\omega_{n}\right)$.

\section{f. Unitarity}

It is instructive to verify that the Floquet scattering matrix we calculated is unitary. Let us, for instance, prove the following unitarity condition: ${ }^{1}$

$$
\begin{aligned}
& \sum_{p=-\infty}^{\infty} S_{F}^{*}(E+p \hbar \Omega, E-m \hbar \Omega) \\
& \quad \times S_{F}(E+p \hbar \Omega, E-n \hbar \Omega)=\delta_{m, n},
\end{aligned}
$$

where $p, m, n$ all are integers. Using Eq. (A3b) and the normalized quantities of Eq. (A21b) and going over to the continuous frequency representation we arrive at the following identity to prove:

$$
\begin{aligned}
& \int_{-\infty}^{\infty} d \omega_{p} S_{\text {out }}^{*}\left(\epsilon+\omega_{p}, \omega_{m}+\omega_{p}\right) S_{\text {out }}\left(\epsilon+\omega_{p}, \omega_{n}+\omega_{p}\right) \\
& =\left(\frac{\hbar \Omega}{\Delta}\right)^{2} \delta\left(\omega_{m}-\omega_{n}\right) .
\end{aligned}
$$

With Eq. (A22a) we get

$$
\begin{aligned}
\int_{-\infty}^{\infty} & d \omega_{p} \frac{\sin \left(\pi\left[\omega_{m}+\omega_{p}\right]\right) \sin \left(\pi\left[\omega_{n}+\omega_{p}\right]\right)}{\pi^{2}\left[\omega_{m}+\omega_{p}\right]\left[\omega_{n}+\omega_{p}\right]} e^{i \pi\left[\omega_{m}-\omega_{n}\right]} \\
& \times\left\{\delta\left(\omega_{m}+\omega_{p}\right)-\frac{\frac{e^{-i 2 \pi\left[\omega_{m}+\omega_{p}\right] t_{-} / \tau}}{1-\omega_{m}-\omega_{p}}+\frac{e^{-i 2 \pi\left[\omega_{m}+\omega_{p}\right] t_{+} / \tau}}{1+\omega_{m}+\omega_{p}}}{\rho\left(\epsilon+\omega_{p}\right) \rho^{*}\left(\epsilon-\omega_{m}\right)}\right\} \\
& \times\left\{\delta\left(\omega_{n}+\omega_{p}\right)-\frac{\frac{e^{i 2 \pi\left[\omega_{n}+\omega_{p}\right] t_{-} / \tau}}{1-\omega_{n}-\omega_{p}}+\frac{e^{i 2 \pi\left[\omega_{n}+\omega_{p}\right] t_{+} / \tau}}{1+\omega_{n}+\omega_{p}}}{\rho^{*}\left(\epsilon+\omega_{p}\right) \rho\left(\epsilon-\omega_{n}\right)}\right\} \\
= & \delta\left(\omega_{m}-\omega_{n}\right) .
\end{aligned}
$$


Here we used the property of the stationary scattering amplitude: $\left|S\left(\epsilon+\omega_{p}\right)\right|^{2}=1$. Next we open the curly brackets,

$$
\begin{aligned}
\int_{-\infty}^{\infty} & \frac{d \omega_{p}}{\left|\rho\left(\epsilon+\omega_{p}\right)\right|^{2}} \frac{\sin \left(\pi\left[\omega_{m}+\omega_{p}\right]\right) \sin \left(\pi\left[\omega_{n}+\omega_{p}\right]\right)}{\pi^{2}\left[\omega_{m}+\omega_{p}\right]\left[\omega_{n}+\omega_{p}\right]}\left\{\xi_{p}+\frac{e^{-i 2 \pi\left[\omega_{m}-\omega_{n}\right] t_{-} / \tau}}{\left(\omega_{m}+\omega_{p}-1\right)\left(\omega_{n}+\omega_{p}-1\right)}+\frac{e^{-i 2 \pi\left[\omega_{m}-\omega_{n}\right] t_{+} / \tau}}{\left(\omega_{m}+\omega_{p}+1\right)\left(\omega_{n}+\omega_{p}+1\right)}\right\} \\
= & \frac{2 \sin \left(\pi\left[\omega_{m}-\omega_{n}\right]\right)}{\pi\left[\omega_{m}-\omega_{n}\right]} \frac{e^{-i 2 \pi\left[\omega_{m}-\omega_{n}\right] t_{-} / \tau}+e^{-i 2 \pi\left[\omega_{m}-\omega_{n}\right] t_{+} / \tau}}{1-\left[\omega_{m}-\omega_{n}\right]^{2}}
\end{aligned}
$$

where

$$
\begin{aligned}
\xi_{p}= & -e^{-i 2 \pi \omega_{p}\left(t_{+}-t_{-}\right) / \tau} \frac{e^{i 2 \pi \omega_{n}\left(t_{-} / \tau\right)} e^{-i 2 \pi \omega_{m}\left(t_{+} / \tau\right)}}{\left(\omega_{n}+\omega_{p}-1\right)\left(\omega_{m}+\omega_{p}+1\right)} \\
& -e^{i 2 \pi \omega_{p}\left(t_{+}-t_{-}\right) / \tau} \frac{e^{i 2 \pi \omega_{n}\left(t_{+} / \tau\right)} e^{-i 2 \pi \omega_{m}\left(t_{-} / \tau\right)}}{\left(\omega_{n}+\omega_{p}+1\right)\left(\omega_{m}+\omega_{p}-1\right)} .
\end{aligned}
$$

Since the time period between the potential steps is much larger than the duration of one turn, $\left|t_{+}-t_{-}\right| \gg \tau$, see Eqs. (A17) and (2), the quantity $\xi_{p}$ oscillates fast as a function of $\omega_{p}$. The terms under the integral over $\omega_{p}$ which are a product of a function that oscillates fast with a smooth function are zero. Hence we can ignore $\xi_{p}$ in Eq. (A26). Physically it means that the emission of an electron at time $t_{-}$has no effect on the emission of a hole at time $t_{+}$. Therefore, one can calculate quantities (current, heat, etc.) caused separately by either electrons or holes. To this end in Eqs. (A22) we remove the part with either $e^{i 2 \pi \omega_{n} t_{+} / \tau}$ or $e^{i 2 \pi \omega_{n} t_{-} / \tau}$, respectively.

To prove Eq. (A26) (without $\xi_{p}$ ) we note that $t_{-}$and $t_{+}$are arbitrary and, therefore, the parts with the factors $e^{-i 2 \pi\left[\omega_{m}-\omega_{n}\right] t_{-} / \tau}$ or $e^{-i 2 \pi\left[\omega_{m}-\omega_{n}\right] t_{+} / \tau}$ have to be considered separately. Therefore we have to show that

$$
\begin{aligned}
\int_{-\infty}^{\infty} & \frac{d \omega_{p}}{\left|\rho\left(\epsilon+\omega_{p}\right)\right|^{2}} \frac{\sin \left(\pi\left[\omega_{m}+\omega_{p}\right]\right) \sin \left(\pi\left[\omega_{n}+\omega_{p}\right]\right)}{\pi^{2}\left[\omega_{m}+\omega_{p}\right]\left[\omega_{n}+\omega_{p}\right]} \\
& \times \frac{1}{\left(\omega_{m}+\omega_{p} \mp 1\right)\left(\omega_{n}+\omega_{p} \mp 1\right)} \\
= & \frac{2 \sin \left(\pi \omega_{q}\right)}{\pi \omega_{q}\left(1-\omega_{q}^{2}\right)}
\end{aligned}
$$

where $\omega_{q}=\omega_{m}-\omega_{n}$. To simplify calculations we do the following: Since $\rho\left(\epsilon+\omega_{p}\right)$, Eq. (A21c), is periodic in $\omega_{p}$ with period 1 , we integrate over one period and sum up contributions from all periods. So we replace

$$
\int_{-\infty}^{\infty} d \omega_{p} \rightarrow \sum_{a=-\infty}^{\infty} \int_{0}^{1} d \omega_{p}^{\prime}, \quad \omega_{p} \rightarrow \omega_{p}^{\prime}+a,
$$

and get

$$
\int_{0}^{1} d \omega_{p}^{\prime} \frac{\Sigma_{q}}{\left|\rho\left(\epsilon+\omega_{p}^{\prime}\right)\right|^{2}}=\frac{2 \sin \left(\pi \omega_{q}\right)}{\pi \omega_{q}\left(1-\omega_{q}^{2}\right)},
$$

where

$$
\begin{aligned}
\Sigma_{q}= & \sum_{a=-\infty}^{\infty} \frac{\sin \left(\pi\left[\omega_{m}+\omega_{p}^{\prime}\right]\right) \sin \left(\pi\left[\omega_{n}+\omega_{p}^{\prime}\right]\right)}{\pi^{2}\left[\omega_{m}+\omega_{p}^{\prime}+a\right]\left[\omega_{n}+\omega_{p}^{\prime}+a\right]} \\
& \times \frac{1}{\left(\omega_{m}+\omega_{p}^{\prime}+a \mp 1\right)\left(\omega_{n}+\omega_{p}^{\prime}+a \mp 1\right)} .
\end{aligned}
$$

To calculate $\Sigma_{q}$ we use the following identity:

$$
\begin{aligned}
\sigma_{2} & \equiv \sum_{a=-\infty}^{\infty} \frac{1}{\left\{(a-\delta)^{2}-\frac{1}{4}\right\}\left\{(a-[x+\delta])^{2}-\frac{1}{4}\right\}} \\
& =\frac{\sin (\pi x)}{x\left(1-x^{2}\right)} \frac{2 \pi}{\cos (\pi \delta) \cos (\pi[x+\delta])},
\end{aligned}
$$

which can be proven with the help of the following text-book sum:

$$
\sigma_{0}(\gamma) \equiv \sum_{a=-\infty}^{\infty} \frac{1}{a+\gamma}=\pi \cot (\pi \gamma)
$$

taken with different arguments,

$$
\begin{aligned}
\sigma_{2}= & \left\{\sigma_{0}\left(-[x+\delta] \pm \frac{1}{2}\right)-\sigma_{0}\left(-\delta \pm \frac{1}{2}\right)\right\} \\
& \times\left\{\frac{2}{x}+\frac{1}{1-x}-\frac{1}{1+x}\right\} .
\end{aligned}
$$

So, in Eq. (A31) we introduce $-\delta \pm 0.5=\omega_{m}+\omega_{p}^{\prime}$ and $-[x+\delta] \pm 0.5=\omega_{n}+\omega_{p}^{\prime}$, use Eq. (A32), and obtain

$$
\Sigma_{q}=\frac{2 \sin \left(\pi \omega_{q}\right)}{\pi \omega_{q}\left(1-\omega_{q}^{2}\right)} .
$$

Since $\Sigma_{q}$ is independent of $\omega_{p}^{\prime}$, we can integrate in Eq. (A30). With $\rho$ given in Eq. (A21c) we get 1. Therefore, the use of Eq. (A33) in Eq. (A30) gives the identity. This completes the proof of Eq. (A24).

\section{APPENDIX B: ZERO-FREQUENCY NOISE POWER}

\section{Quantized noise of a single source}

For a moment we switch off, say, source $B$. Now we use $S_{\text {out }, p-n}^{B}\left(E_{p}\right)=\delta_{p, n}$ in Eq. (16) and reduce Eq. (15) to

$$
\begin{aligned}
\mathcal{P}_{34}= & -\frac{e^{2}}{h}\left(1-T_{C}\right) T_{C} \int_{0}^{\infty} d E \sum_{n=-\infty}^{\infty}\left\{f\left(E_{-n}\right)-f(E)\right\}^{2} \\
& \times\left|S_{\text {out }, n}^{A}(E)\right|^{2} .
\end{aligned}
$$

Here $T_{C}=\left|t_{C}\right|^{2}$ is the probability for an electron to pass through QPC $C$ connecting the two waveguides as shown in Fig. 3. In above equation we changed $n \rightarrow-n$ and used both the following relation $r_{C} t_{C}^{*}=-r_{C}^{*} t_{C}$ and the unitarity 
condition for $S_{\text {out }}^{A}$ :

$$
\sum_{p=-\infty}^{\infty} S_{\text {out }, p-m}^{A *}\left(E_{p}\right) S_{\text {out }, p-n}^{A}\left(E_{p}\right)=\delta_{n, m},
$$

which follows directly from Eq. (A23).

Next with the quantities introduced in Eqs. (A21) and with Eq. (A22b) we rewrite Eq. (B1) as follows:

$$
\begin{aligned}
\mathcal{P}_{34}^{n a}= & -\mathcal{P}_{0} \int_{-\infty}^{\infty} d \epsilon \int_{-\infty}^{\infty} d \omega_{n} \frac{\left\{f\left(\epsilon-\omega_{n}\right)-f(\epsilon)\right\}^{2}}{\left|\rho\left(\epsilon-\omega_{n}\right)\right|^{2}|\rho(\epsilon)|^{2}} \\
& \times \frac{\sin ^{2}\left(\pi \omega_{n}\right)}{\pi^{2} \omega_{n}^{2}}\left\{\frac{1}{\left(1-\omega_{n}\right)^{2}}+\frac{1}{\left(1+\omega_{n}\right)^{2}}\right\} .
\end{aligned}
$$

Here we have dropped the terms $\sim \exp \left(i 2 \pi\left[\epsilon+\omega_{n}\right]\left(t_{-}^{A}-\right.\right.$ $\left.\left.t_{+}^{A}\right) / \tau\right)$ as noncontributing. Since $\left|t_{-}^{A}-t_{+}^{B}\right| / \tau \gg 1$ these terms oscillate fast in both $\epsilon$ and $\omega_{n}$. Therefore, they are nullified after the integration. The upper index " $n a$ " emphasizes that this equation is for the time-dependent potential $U(t)$, Eq. (A8), leading to nonadiabatic emission of particles. Note that the parts proportional $1 /\left(1-\omega_{n}\right)^{2}$ and $1 /\left(1+\omega_{n}\right)^{2}$ correspond to an electron and a hole contributions, respectively.

With $\rho(\epsilon)$, Eq. (A21c), in the limit of $T \rightarrow 0$ we represent the density of states (normalized to $\Delta$ ) as a sum of Breit-
Wigner resonances each of unit area:

$$
\begin{aligned}
\frac{1}{|\rho(\epsilon)|^{2}} & =\sum_{\ell=-\infty}^{\infty} \frac{g / \pi}{(\epsilon+0.5-\ell)^{2}+g^{2}}, \\
\frac{1}{\left|\rho\left(\epsilon-\omega_{n}\right)\right|^{2}} & =\sum_{n=-\infty}^{\infty} \frac{g / \pi}{\left(\epsilon-\omega_{n}+0.5-n\right)^{2}+g^{2}},
\end{aligned}
$$

with $g=T /(4 \pi)$ a width (normalized to $\Delta$ ).

Integrating over $\omega_{n}$, we take into account that the integrand has narrow peaks at the integers $\omega_{n}=\ell-n$, where the sinus is zero. Therefore, to leading order in $g \ll 1$ what matters is only $\ell=n, \ell=n \pm 1$ when the zeros of the denominator cancel the zero of $\sin \left(\pi \omega_{n}\right)$. Because of the difference of the Fermi distribution functions, the term with $\ell=n$ does not contribute. In addition, if the temperature is much lower then the level spacing, we can approximate $f(\epsilon) \approx \theta(-\epsilon)$. Using this, we find that only the pairs $\ell=1, n=0$ (an electron emission) and $\ell=0, n=1$ (a hole emission) contribute. Therefore, we arrive at

$$
\mathcal{P}_{34}^{n a}=-2 \mathcal{P}_{0},
$$

announced already in Eq. (17).

\section{Shot-noise suppression effect}

If both sources $A$ and $B$ are switched on then

$$
\begin{aligned}
\mathcal{P}_{34}= & -\mathcal{P}_{0} \frac{\Delta^{4}}{(\hbar \Omega)^{4}} \int_{-\infty}^{\infty} d \epsilon \int_{-\infty}^{\infty} d \omega_{m} \int_{-\infty}^{\infty} d \omega_{n}\left\{f\left(\epsilon+\omega_{n}\right)-f\left(\epsilon+\omega_{m}\right)\right\}^{2} \\
& \times \operatorname{Re} \int_{-\infty}^{\infty} d \omega_{p} e^{i 2 \pi \omega_{p}(\delta \tau / \tau)} S_{\text {out }}^{A *}\left(\epsilon,-\omega_{n}\right) S_{\text {out }}^{B}\left(\epsilon,-\omega_{m}\right) S_{\text {out }}^{B *}\left(\epsilon+\omega_{p}, \omega_{p}-\omega_{m}\right) S_{\text {out }}^{A}\left(\epsilon+\omega_{p}, \omega_{p}-\omega_{n}\right),
\end{aligned}
$$

where Re indicates the real part of an expression and

$$
\delta \tau=\tau_{L^{A}}-\tau_{L^{B}} .
$$

Our aim here is to analyze how the shot noise depends on the difference of times when particles emitted by the different sources pass QPC $C$. This difference depends on both the time when the particles were emitted and the time necessary for them to propagate to QPC $C$. Without loss of generality we assume that cavities $A$ and $B$ emit particles of the same kind (electrons or holes) at the same time. Therefore, in this subsection we use

$$
S_{\text {out }}^{A}=S_{\text {out }}^{B} \equiv S_{\text {out }} .
$$

Thus $\delta \tau$ alone determines the difference of times when the particles pass QPC $C$ : If $\delta \tau \gg \tau_{D}$ the particles pass QPC $C$ independently, whereas if $\delta \tau=0$ they will collide.

\section{a. Independent particles}

\section{If}

$$
\delta \tau \gg \tau_{D}
$$

then we show that

$$
\mathcal{P}_{34}=-4 \mathcal{P}_{0},
$$

i.e., each particle contributes the same value $-\mathcal{P}_{0}$.
To arrive at Eq. (B10) we first represent the Fermi functions difference in Eq. (B6) as

$$
\begin{aligned}
\left\{f\left(\epsilon+\omega_{n}\right)-f\left(\epsilon+\omega_{m}\right)\right\}^{2} \\
=\left\{f\left(\epsilon+\omega_{n}\right)-f(\epsilon)\right\}^{2}+\left\{f(\epsilon)-f\left(\epsilon+\omega_{m}\right)\right\}^{2} \\
\quad+2\left\{f\left(\epsilon+\omega_{n}\right)-f(\epsilon)\right\}\left\{f(\epsilon)-f\left(\epsilon+\omega_{m}\right)\right\} .
\end{aligned}
$$

Then, for instance, with the first term on the right-hand side of Eq. (B11) and with Eq. (B8) we can integrate out $\omega_{m}$ in Eq. (B6). Next we use the unitarity condition ${ }^{1}$

$$
\begin{aligned}
& \int_{-\infty}^{\infty} d \omega_{m} S_{\text {out }}^{*}\left(\epsilon+\omega_{p}, \omega_{p}-\omega_{m}\right) S_{\text {out }}\left(\epsilon+\omega_{q}, \omega_{q}-\omega_{m}\right) \\
& \quad=\left(\frac{\hbar \Omega}{\Delta}\right)^{2} \delta\left(\omega_{p}-\omega_{q}\right)
\end{aligned}
$$

complementary to Eq. (A24) and get $\delta\left(\omega_{p}\right)$. After that we integrate out $\omega_{p}$, and arrive at an equation similar to Eq. (B1), which is shown to be equal to $-2 \mathcal{P}_{0}$, see Eq. (B5). The same procedure with the second term on the right-hand side of Eq. (B11) results in a second contribution $-2 \mathcal{P}_{0}$. To prove Eq. (B10) we have to show additionally that what remains in 
Eq. (B6) is zero,

$$
\mathcal{P}_{34}^{\text {rest }}=2 \mathcal{P}_{0} \int_{-\infty}^{\infty} d \epsilon \operatorname{Re} \int_{-\infty}^{\infty} d \omega_{p} e^{i 2 \pi \omega_{p} \delta \tau / \tau}\left|J\left(\epsilon, \omega_{p}\right)\right|^{2},
$$

where

$$
\begin{aligned}
J\left(\epsilon, \omega_{p}\right)= & \frac{\Delta^{2}}{(\hbar \Omega)^{2}} \int_{-\infty}^{\infty} d \omega_{n}\left\{f\left(\epsilon+\omega_{n}\right)-f(\epsilon)\right\} \\
& \times S_{\text {out }}^{*}\left(\epsilon,-\omega_{n}\right) S_{\text {out }}\left(\epsilon+\omega_{p}, \omega_{p}-\omega_{n}\right) .
\end{aligned}
$$

To show this we note that $|\delta \tau| \gg \tau$ as it follows from Eqs. (B9) and (2) for $T \ll 1$. We see that the integrand in Eq. (B13) oscillates fast with $\omega_{p}$ and, therefore, the integral over $\omega_{p}$ is zero, $\mathcal{P}_{34}^{\text {rest }}=0$.

\section{b. Colliding particles}

If the particles collide at QPC $C$,

$$
\delta \tau=0,
$$

then the noise is zero,

$$
\mathcal{P}_{34}=0 .
$$

This follows directly from Eq. (B6), where we use Eqs. (B8) and (A24) and integrate over $\omega_{p}$. As a result we find $\delta\left(\omega_{n}-\right.$ $\omega_{m}$ ), which in turn vanishes after the integration, say, over $\omega_{n}$, due to the different Fermi functions.

We emphasize that both Eqs. (B10) and (B15) were obtained without any reference to the condition of emission.

\section{c. Partial overlap of wave packets}

Now we analyze how the noise depends on the overlap of particles at QPC $C$. The overlap is a function of the time delay $\delta \tau \sim \tau_{D}$. We use Eqs. (B8) and (A22b) in Eq. (B6), which after the substitutions $\omega_{p} \rightarrow \omega_{p}-\epsilon, \omega_{n / m} \rightarrow-\omega_{n / m}-\epsilon$ can be cast into the following form:

$$
\begin{aligned}
\mathcal{P}_{34}^{n a}= & -\mathcal{P}_{0} \int_{-\infty}^{\infty} d \omega_{n} \int_{-\infty}^{\infty} d \omega_{m}\left|J\left(\omega_{n}, \omega_{m}\right)\right|^{2} \\
& \times\left\{f\left(-\omega_{n}\right)-f\left(-\omega_{m}\right)\right\}^{2},
\end{aligned}
$$

where

$$
\begin{aligned}
& J\left(\omega_{n}, \omega_{m}\right) \\
& =\int_{-\infty}^{\infty} d \omega_{p} e^{i 2 \pi \omega_{p} \delta \tau / \tau} \frac{\sin \left(\pi\left[\omega_{p}+\omega_{n}\right]\right)}{\pi\left[\omega_{p}+\omega_{n}\right]} \frac{\sin \left(\pi\left[\omega_{p}+\omega_{m}\right]\right)}{\pi\left[\omega_{p}+\omega_{m}\right]} \\
& \quad \times\left\{\delta\left(\omega_{p}+\omega_{n}\right)-\frac{\frac{e^{i 2 \pi\left[\omega_{p}+\omega_{n}\right] t_{-} / \tau}}{\left(1-\omega_{p}-\omega_{n}\right)}+\frac{e^{i 2 \pi\left[\omega_{p}+\omega_{n}\right] t_{+} / \tau}}{\left(1+\omega_{p}+\omega_{n}\right)}}{\rho^{*}\left(\omega_{p}\right) \rho\left(-\omega_{n}\right)}\right\} \\
& \quad \times\left\{\delta\left(\omega_{p}+\omega_{m}\right)-\frac{\frac{e^{i 2 \pi\left[\omega_{p}+\omega_{m}\right] t_{t} / \tau}}{\left(1-\omega_{p}-\omega_{m}\right)}+\frac{e^{i 2 \pi\left[\omega_{p}+\omega_{m}\right]_{+} / \tau}}{\left(1+\omega_{p}+\omega_{m}\right)}}{\rho\left(\omega_{p}\right) \rho^{*}\left(-\omega_{m}\right)}\right\} .
\end{aligned}
$$

To simplify the equation above we note the following. First, the term $\delta\left(\omega_{m}-\omega_{n}\right)$ does not contribute to Eq. (B16).
Second, since $\left|t_{-}-t_{+}\right| \sim \mathcal{T} \gg \tau$ then any term containing $\exp \left\{\omega_{p}\left[t_{-}-t_{+}\right] / \tau\right\}$ results in zero after the integration over $\omega_{p}$. Furthermore, after simple algebra we find

$$
J\left(\omega_{n}, \omega_{m}\right)=\frac{\mathcal{A}_{-} e^{i 2 \pi \omega_{q} t_{-} / \tau}+\mathcal{A}_{+} e^{i 2 \pi \omega_{q} t_{+} / \tau}}{\rho^{*}\left(-\omega_{m}\right) \rho\left(-\omega_{n}\right)} \frac{\sin \left(\pi \omega_{q}\right)}{\pi \omega_{q}},
$$

where $\omega_{q}=\omega_{n}-\omega_{m}$ and

$$
\mathcal{A}_{\mp}=\mp \frac{e^{-i 2 \pi \omega_{n} \delta \tau / \tau}}{\omega_{q} \pm 1} \pm \frac{e^{-i 2 \pi \omega_{m} \delta \tau / \tau}}{\omega_{q} \mp 1}+\hat{\mathcal{A}}_{\mp}
$$

with

$$
\begin{aligned}
\hat{\mathcal{A}}_{\mp}= & \frac{\pi \omega_{q}}{\sin \left(\pi \omega_{q}\right)} \int_{-\infty}^{\infty} d \omega_{p} \frac{e^{i 2 \pi \omega_{p} \delta \tau / \tau}}{\pi^{2}\left|\rho\left(\omega_{p}\right)\right|^{2}} \\
& \times \frac{\sin \left(\pi\left[\omega_{p}+\omega_{n}\right]\right) \sin \left(\pi\left[\omega_{p}+\omega_{m}\right]\right)}{\left[\omega_{p}+\omega_{n}\right]\left(\omega_{p}+\omega_{n} \mp 1\right)\left[\omega_{p}+\omega_{m}\right]\left(\omega_{p}+\omega_{m} \mp 1\right)} .
\end{aligned}
$$

We evaluate the last integral in the same way as we evaluated the integral in Eq. (A28):

$$
\hat{\mathcal{A}}_{\mp}=\frac{2}{1-\omega_{q}^{2}} \int_{0}^{1} d \omega_{p}^{\prime} \frac{e^{i 2 \pi \omega_{p}^{\prime} \delta \tau / \tau}}{\left|\rho\left(\omega_{p}^{\prime}\right)\right|^{2}} .
$$

Factorizing the original integral into the product of the integral over a single period and the sum over different periods we used the following prescription: The quantity $\hat{A}_{\mp}$ is a continuous function of $\delta \tau$ and it is changed only a little on the scale of $\tau$, which is the smallest time scale in the problem and in many cases we put it to be zero, $\tau \rightarrow 0$. Therefore, we always consider $\delta \tau / \tau \gg 1$ to be, for instance, an integer. As a consequence the integral in Eq. (B21) is the same for any period of the density of states $1 /\left|\rho\left(\omega_{p}\right)\right|^{2}$. Using Eq. (B4) we finally arrive at

$$
\hat{\mathcal{A}}_{\mp}=\frac{2 e^{-|\delta \tau| / 2 \tau_{D}}}{1-\omega_{q}^{2}} .
$$

To proceed we calculate $\left|J\left(\omega_{n}, \omega_{m}\right)\right|^{2}$ and keep only the terms with factors $\left|\mathcal{A}_{-}\right|^{2}$ and $\left|\mathcal{A}_{+}\right|^{2}$. All other terms, which have fast oscillating factors $e^{i 2 \pi \omega_{q} t_{F} / \tau}$, will be nullified after the integration over $\omega_{n / m}$ in Eq. (B16). Thus,

$$
\begin{aligned}
\mathcal{P}_{34}^{n a}= & -\mathcal{P}_{0} \int_{-\infty}^{\infty} \frac{d \omega_{n}}{\left|\rho\left(\omega_{n}\right)\right|^{2}} \int_{-\infty}^{\infty} \frac{d \omega_{m}}{\left|\rho\left(\omega_{m}\right)\right|^{2}} \\
& \times\left\{f\left(\omega_{n}\right)-f\left(\omega_{m}\right)\right\}^{2} \frac{4 \sin ^{2}\left(\pi \omega_{q}\right)}{\pi^{2} \omega_{q}^{2}\left(1-\omega_{q}^{2}\right)^{2}} \\
& \times\left\{\mathcal{B}\left(\omega_{q}\right)-4 e^{-|\delta \tau| / 2 \tau_{D}} \cos \left(2 \pi \omega_{n} \frac{\delta \tau}{\tau}\right)\right\},
\end{aligned}
$$


where

$$
\mathcal{B}\left(\omega_{q}\right)=\omega_{q}^{2}+1+2 e^{-|\delta \tau| / \tau_{D}}-\left[\omega_{q}^{2}-1\right] \cos \left(2 \pi \omega_{q} \frac{\delta \tau}{\tau}\right) .
$$

Note in Eq. (B23) we changed the sign, $\omega_{n / m} \rightarrow-\omega_{n / m}$, compared to Eq. (B16). In addition we used the symmetry with respect to $\omega_{n}$ and $\omega_{m}$ and replaced $\cos \left(2 \pi \omega_{n} \delta \tau / \tau\right)+$ $\cos \left(2 \pi \omega_{m} \delta \tau / \tau\right)$ by $2 \cos \left(2 \pi \omega_{n} \delta \tau / \tau\right)$.

Let us first evaluate the part of Eq. (B23) with $\mathcal{B}\left(\omega_{q}\right)$. We use Eq. (B4). In the leading order in $g \rightarrow 0$ and at small temperatures, $f\left(\omega_{n}\right) \approx \theta\left(-\omega_{n}\right)$, we find that only $\omega_{q}= \pm 1$ is relevant. Using $\mathcal{B}( \pm 1)=2\left[1+\exp \left(-|\delta \tau| / \tau_{D}\right)\right]$ we calculate the corresponding contribution

$$
\mathcal{P}_{34}^{n a, 1}=-4 \mathcal{P}_{0}\left\{1+e^{-|\delta \tau| / \tau_{D}}\right\} .
$$

Similarly we evaluate the remaining part,

$$
\begin{aligned}
\mathcal{P}_{34}^{n a, 2} & =8 e^{-|\delta \tau| / 2 \tau_{D}} \mathcal{P}_{0} \int_{-0.5}^{0.5} d \omega_{n} \frac{g / \pi \cos \left(2 \pi \omega_{n} \frac{\delta \tau}{\tau}\right)}{\omega_{n}^{2}+g^{2}} \\
& =8 e^{-|\delta \tau| / \tau_{D}} \mathcal{P}_{0} .
\end{aligned}
$$

Summing up Eqs. (B25) and (B26) we arrive at

$$
\mathcal{P}_{34}^{n a}=-4 \mathcal{P}_{0}\left\{1-e^{-|\delta \tau| / \tau_{D}}\right\} .
$$

We see that at $|\delta \tau| \rightarrow \infty$, we recover the independent particle case, see Eq. (B10), whereas at $\delta \tau=0$ the zero-noise result for colliding particles is recovered; see Eq. (B15). If electrons and holes have different time delays we arrive at Eq. (18).

\section{APPENDIX C: TWO-PARTICLE EMITTER}

Let two cavities be coupled to the same edge state, a distance $L$ away from each other (see Fig. 2). We assume that the cavities emit particles at different times but are otherwise identical.

\section{Scattering amplitude}

The elements of the Floquet scattering matrix $\hat{S}_{F}^{(2)}$ of the two-cavity system are expressed in terms of the elements of the Floquet scattering matrices of the cavities, $\hat{S}_{F}^{A}$ and $\hat{S}_{F}^{B}$, in the following way: ${ }^{1}$

$$
S_{F}^{(2)}\left(E, E_{n}\right)=\sum_{\ell=-\infty}^{\infty} S_{F}^{B}\left(E, E_{\ell}\right) e^{i \varphi_{L}(E)} e^{i \ell \Omega \tau_{L}} S_{F}^{A}\left(E_{\ell}, E_{n}\right),
$$

where $\varphi_{L}(E)$ is the phase factor describing free propagation between the cavities, $\tau_{L}$ is the time of flight from $A$ to $B$. Using Eqs. (A21) and introducing $S_{\text {out }}^{j}$ we rewrite

$$
S_{\text {out }}^{(2)}\left(\epsilon,-\omega_{n}\right)=\frac{\Delta}{\hbar \Omega} \int d \omega_{\ell} e^{i \varphi_{L}(\epsilon)} e^{i 2 \pi \omega_{\ell} \tau_{L} / \tau} S_{\text {out }}^{B}\left(\epsilon,-\omega_{\ell}\right) S_{\text {out }}^{A}\left(\epsilon+\omega_{\ell}, \omega_{\ell}-\omega_{n}\right) .
$$

We simplify the above equation in two important cases: (i) if the cavities emit an electron and a hole at close times, and (ii) if they emit two electrons at close times. We use $S_{\text {out }}^{j}$, Eq. (A22b), with $t_{\mp}$ replaced by $t_{\mp}^{j}$. We will use the upper indices “eh" and "ee" to distinguish quantities related to these cases.

\section{a. Electron-hole emission}

We keep the terms with $t_{-}^{A}$ and $t_{+}^{B}$ in equations for $S_{\text {out }}^{A}$ and $S_{\text {out }}^{B}$, respectively, and find

$$
\begin{aligned}
S_{\text {out }}^{(2) e h}\left(\epsilon,-\omega_{n}\right)= & e^{i\left[\psi(\epsilon)+\pi \omega_{n}\right]} \frac{\hbar \Omega}{\Delta} \frac{\rho^{*}(\epsilon)}{\rho(\epsilon)} \frac{\rho^{*}\left(\epsilon+\omega_{n}\right)}{\rho\left(\epsilon+\omega_{n}\right)} e^{-i 2 \pi \omega_{n} t_{-}^{A} / \tau} \\
& \times\left\{\frac{\delta S_{\text {out }}^{(2) h}\left(\epsilon,-\omega_{n}\right)}{\rho^{*}(\epsilon) \rho^{*}\left(\epsilon+\omega_{n}\right)}+\delta\left(\omega_{n}\right)+\frac{\sin \left(\pi \omega_{n}\right)}{\pi \omega_{n}}\left[\frac{e^{i 2 \pi \omega_{n} \delta t^{e h} / \tau} /\left(\omega_{n}-1\right)}{\rho^{*}(\epsilon) \rho\left(\epsilon+\omega_{n}\right)}-\frac{1 /\left(\omega_{n}+1\right)}{\rho^{*}\left(\epsilon+\omega_{n}\right) \rho(\epsilon)}\right]\right\},
\end{aligned}
$$

where $\psi(\epsilon)=2 \theta_{r}+2 \phi\left(U_{0} / \Delta, \epsilon\right)+\varphi_{L}(\epsilon)$ and

$$
\delta t^{e h}=t_{-}^{A}+\tau_{d}-t_{+}^{B},
$$

and

$$
\delta S_{\text {out }}^{(2) e h}\left(\epsilon,-\omega_{n}\right)=\int d \omega_{\ell} \frac{\sin \left(\pi \omega_{\ell}\right)}{\pi \omega_{\ell}} \frac{\sin \left(\pi\left[\omega_{\ell}-\omega_{n}\right]\right)}{\pi\left[\omega_{\ell}-\omega_{n}\right]} \frac{\exp \left\{i 2 \pi \omega_{\ell} \frac{\delta t^{e h}}{\tau}\right\}}{\left(\omega_{\ell}-1\right)\left(\omega_{\ell}-\omega_{n}-1\right) \rho^{2}\left(\epsilon+\omega_{\ell}\right)} .
$$


Here in $\left.\exp _{\{} i 2 \pi \omega_{\ell} \delta t^{e h} / \tau\right\}$ we have neglected $\tau$ compared to $\delta t^{e h}$. To simplify further we expand $1 / \rho^{2}$ into the sum of the Breit-Wigner resonances,

$$
\frac{1}{\rho^{2}\left(\epsilon+\omega_{\ell}\right)}=\sum_{a=-\infty}^{\infty} \frac{-g / \pi}{\left(\epsilon+\omega_{\ell}+0.5-a+i g\right)^{2}},
$$

and integrate out $\omega_{\ell}$ as we already did. As a result we obtain

$$
\delta S_{\text {out }}^{(2) e h}\left(\epsilon,-\omega_{n}\right)=\theta\left(-\delta t^{e h}\right) e^{-\left|\delta t^{e h}\right| / 2 \tau_{D}} \frac{\delta t^{e h}}{\tau_{D}} e^{-i 2 \pi \epsilon \delta t^{e h} / \tau} \frac{2 \sin \left(\pi \omega_{n}\right)}{\pi \omega_{n}\left(\omega_{n}^{2}-1\right)} .
$$

Interestingly, this term vanishes at $\delta t^{e h}=0$, when an electron and a hole are emitted simultaneously, as well as at $\left|\delta t^{e h}\right| \gg \tau_{D}$, when they are emitted independently.

\section{b. Two-electron emission}

Keeping the terms with $t_{-}^{A}$ and $t_{-}^{B}$ in equations for $S_{\text {out }}^{A}$ and $S_{\text {out }}^{B}$, respectively, we calculate

$$
\begin{aligned}
S_{\text {out }}^{(2) e e}\left(\epsilon,-\omega_{n}\right)= & e^{i\left[\psi(\epsilon)+\pi \omega_{n}\right]} \frac{\hbar \Omega}{\Delta} \frac{\rho^{*}(\epsilon)}{\rho(\epsilon)} \frac{\rho^{*}\left(\epsilon+\omega_{n}\right)}{\rho\left(\epsilon+\omega_{n}\right)} e^{-i 2 \pi \omega_{n} t_{-}^{A} / \tau} \\
& \times\left\{\frac{\delta S_{\text {out }}^{(2) e e}\left(\epsilon,-\omega_{n}\right)}{\rho^{*}(\epsilon) \rho^{*}\left(\epsilon+\omega_{n}\right)}+\delta\left(\omega_{n}\right)-\frac{\sin \left(\pi \omega_{n}\right)}{\pi \omega_{n}\left(\omega_{n}+1\right)}\left[\frac{e^{i 2 \pi \omega_{n}\left(\delta t^{e e} / \tau\right)}}{\rho^{*}(\epsilon) \rho\left(\epsilon+\omega_{n}\right)}+\frac{1}{\rho^{*}\left(\epsilon+\omega_{n}\right) \rho(\epsilon)}\right]\right\}, \\
& \delta S_{\text {out }}^{(2) e e}\left(\epsilon,-\omega_{n}\right)=\theta\left(-\delta t^{e e}\right) e^{-\left|\delta t^{e e}\right| / 2 \tau_{d}} \frac{\delta t^{e e}}{\tau_{D}} e^{-i 2 \pi \epsilon \delta t^{e e} / \tau} \frac{2 \sin \left(\pi \omega_{n}^{\prime}\right)}{\pi \omega_{n}^{\prime}\left(1-\omega_{n}^{\prime 2}\right)},
\end{aligned}
$$

where $\omega_{n}^{\prime}=\omega_{n}+1$ and

$$
\delta t^{e e}=t_{-}^{A}+\tau_{d}-t_{-}^{B} .
$$

The term $\delta S_{\text {out }}^{(2) e e}\left(\epsilon,-\omega_{n}\right)$ is irrelevant in both the case of emission of independent electrons, $\left|\delta t^{e e}\right| \gg \tau_{D}$, and in the case of emission of a pair of electrons, $\delta t^{e e}=0$.

\section{2. dc heat flow}

The excess energy, i.e., the energy over the Fermi energy, carried out by the particles emitted during each period can be calculated as a dc heat flow $I_{d c}^{Q}$, which is expressed in terms of the Floquet scattering amplitude as follows : $:^{1,46}$

$$
\begin{aligned}
I_{d c}^{Q}= & \frac{\Delta^{3}}{h \hbar \Omega} \int_{-\infty}^{\infty} d \omega_{n} \int_{-\infty}^{\infty} \epsilon d \epsilon\left\{f\left(\epsilon+\omega_{n}\right)-f(\epsilon)\right\} \\
& \times\left|S_{\text {out }}^{(2)}\left(\epsilon,-\omega_{n}\right)\right|^{2} .
\end{aligned}
$$

We use this equation to analyze different conditions of emission.

\section{a. Emission of independent particles}

We use Eq. (C3) at $\delta t^{e h} \gg \tau_{D}$ in Eq. (C11) and obtain

$$
\begin{aligned}
I_{d c}^{Q}= & \frac{\Delta}{\mathcal{T}} \int_{-\infty}^{\infty} d \omega_{n} \int_{-\infty}^{\infty} \epsilon d \epsilon \frac{f\left(\epsilon+\omega_{n}\right)-f(\epsilon)}{|\rho(\epsilon)|^{2}\left|\rho\left(\epsilon+\omega_{n}\right)\right|^{2}} \\
& \times \frac{\sin ^{2}\left(\pi \omega_{n}\right)}{\pi^{2} \omega_{n}^{2}}\left\{\frac{1}{\left(\omega_{n}-1\right)^{2}}+\frac{1}{\left(\omega_{n}+1\right)^{2}}\right\} .
\end{aligned}
$$

Here we dropped a term proportional to $\exp \left(i 2 \pi \omega_{n} \delta t^{e h} / \tau\right)$ since it is oscillating fast and hence vanishes after the integration over $\omega_{n}$. To continue we use Eq. (B4) to integrate over $\omega_{n}$ and over $\epsilon$ and find

$$
I_{d c}^{Q}=\frac{\Delta}{\mathcal{T}}
$$

Note that both terms in the curly brackets in Eq. (C12) contribute equally. The first one corresponds to a hole emission and the second one corresponds to an electron emission. Therefore, each particle carries an energy $\mathcal{E}_{n a}=\Delta / 2$, see Eq. (25). The same answer, Eq. (C13), is obtained if we use Eq. (C8) at $\delta t^{e e} \gg \tau_{D}$.

Notice that since both cavities together emit four particles per period, two electrons and two holes, the total dc heat current is twice as large as $I_{d c}^{Q}$, Eq. (C13).

\section{b. Electron-hole pair emission}

If $\delta t^{e h}=0$, then Eq. (C11) with $S_{\text {out }}^{(2) e h}$ from Eq. (C3) gives

$$
\begin{aligned}
I_{d c}^{Q}= & \frac{\Delta}{\mathcal{T}} \int_{-\infty}^{\infty} d \omega_{n} \int_{-\infty}^{\infty} \epsilon d \epsilon \frac{f\left(\epsilon+\omega_{n}\right)-f(\epsilon)}{|\rho(\epsilon)|^{2}\left|\rho\left(\epsilon+\omega_{n}\right)\right|^{2}} \\
& \times \frac{\sin ^{2}\left(\pi \omega_{n}\right)}{\pi^{2} \omega_{n}^{2}}\left\{\frac{1}{\left(\omega_{n}-1\right)^{2}}+\frac{1}{\left(\omega_{n}+1\right)^{2}}-\frac{2 \xi\left(\epsilon, \omega_{n}\right)}{\omega_{n}^{2}-1}\right\},
\end{aligned}
$$

where

$$
\xi\left(\epsilon, \omega_{n}\right)=\operatorname{Re} \frac{\rho^{*}\left(\epsilon+\omega_{n}\right) \rho(\epsilon)}{\rho\left(\epsilon+\omega_{n}\right) \rho^{*}(\epsilon)} .
$$


Evaluating integrals over $\epsilon$ and $\omega_{n}$ at $g \rightarrow 0$ along the same lines as before we find that the term proportional to $\xi\left(\epsilon, \omega_{n}\right)$ does not contribute. Hence, Eq. (C13) remains valid even if an electron and a hole are emitted simultaneously. Therefore, there is no re-absorption ${ }^{27,28}$ under the nonadiabatic emission condition.

\section{c. Electron-electron pair emission}

We substitute Eq. (C8) into Eq. (C11) and find at $\delta t^{e e}=0$ :

$$
\begin{aligned}
I_{d c}^{Q, e e}= & \frac{\Delta}{\mathcal{T}} \int_{-\infty}^{\infty} d \omega_{n} \int_{-\infty}^{\infty} \epsilon d \epsilon \frac{f\left(\epsilon+\omega_{n}\right)-f(\epsilon)}{|\rho(\epsilon)|^{2}\left|\rho\left(\epsilon+\omega_{n}\right)\right|^{2}} \\
& \times \frac{2 \sin ^{2}\left(\pi \omega_{n}\right)\left[1+\xi\left(\epsilon, \omega_{n}\right)\right]}{\pi^{2} \omega_{n}^{2}\left(\omega_{n}+1\right)^{2}} .
\end{aligned}
$$

With $1 /|\rho(\epsilon)|^{2}$ given by Eq. (B4) we calculate at $g \rightarrow 0$ :

$$
I_{d c}^{Q, e e}=\frac{2 \Delta}{\mathcal{T}} .
$$

We see that the energy carried by the two-electron pair, $\mathcal{E}_{n a}^{e e}=2 \Delta$, is as twice as large as the energy, $2 \mathcal{E}_{n a}=\Delta$, carried by two electrons emitted separately. This energy enhancement is due to the Pauli exclusion principle which forbids that the two electrons emitted simultaneously have the same energy.

The doubling of the work done by the two-particle source when it emits two-electron pairs is a peculiar feature common to both adiabatic ${ }^{28}$ and nonadiabatic, Eq. (C17), conditions of emission.
${ }^{1}$ M. V. Moskalets, Scattering Matrix Approach to Non-stationary Quantum Transport (Imperial College Press, London, 2011).

${ }^{2}$ G. Fève, A. Mahé, J.-M. Berroir, T. Kontos, B. Plaçais, D. C. Glattli, A. Cavanna, B. Etienne, and Y. Jin, Science 316, 1169 (2007).

${ }^{3}$ M. D. Blumenthal, B. Kaestner, L. Li, S. Giblin, T. J. B. M. Janssen, M. Pepper, D. Anderson, G. Jones, and D. A. Ritchie, Nat. Phys. 3, 343 (2007).

${ }^{4}$ N. Maire, F. Hohls, B. Kaestner, K. Pierz, H. W. Schumacher, and R. J. Haug, Appl. Phys. Lett. 92, 082112 (2008).

${ }^{5}$ A. Mahé, F. D. Parmentier, E. Bocquillon, J.-M. Berroir, D. C. Glattli, T. Kontos, B. Plaçais, G. Fève, A. Cavanna, and Y. Jin, Phys. Rev. B 82, 201309 (2010).

${ }^{6}$ E. Bocquillon, F. D. Parmentier, C. Grenier, J.-M. Berroir, P. Degiovanni, D. C. Glattli, B. Plaçais, A. Cavanna, Y. Jin, and G. Fève, Phys. Rev. Lett. 108, 196803 (2012).

${ }^{7}$ L. Fricke, M. Wulf, B. Kaestner, V. Kashcheyevs, J. Timoshenko, P. Nazarov, F. Hohls, P. Mirovsky, B. Mackrodt, R. Dolata, T. Weimann, K. Pierz, and H. W. Schumacher, arXiv:1211.1781.

${ }^{8}$ Ch. Grenier, R. Hervé, E. Bocquillon, F. D. Parmentier, B. Plaçais, J. M. Berroir, G. Fève, and P. Degiovanni, New J. Phys. 13, 093007 (2011).

${ }^{9}$ Ch. Grenier, R. Hervé, G. Fève, and P. Degiovanni, Mod. Phys. Lett. B 25, 1053 (2011).

${ }^{10}$ G. Haack, M. Moskalets, and M. Büttiker, arXiv:1212.0088.

${ }^{11}$ A. Fujiwara, K. Nishiguchi, and Y. Ono, Appl. Phys. Lett. 92, 042102 (2008).

${ }^{12}$ B. Kaestner, V. Kashcheyevs, G. Hein, K. Pierz, U. Siegner, and H. W. Schumacher, Appl. Phys. Lett. 92, 192106 (2008).

${ }^{13}$ S. J. Wright, A. L. Thorn, M. D. Blumenthal, S. P. Giblin, M. Pepper, T. J. B. M. Janssen, M. Kataoka, J. D. Fletcher, G. A. C. Jones, C. A. Nicoll, G. Gumbs, and D. A. Ritchie, J. Appl. Phys. 109, 102422 (2011).

${ }^{14}$ C. Leicht, P. Mirovsky, B. Kaestner, F. Hohls, V. Kashcheyevs, E. V. Kurganova, U. Zeitler, T. Weimann, K. Pierz, and H. W. Schumacher, Semicond. Sci. Technol. 26, 055010 (2011).

${ }^{15}$ J. D. Fletcher, M. Kataoka, H. Howe, M. Pepper, P. See, S. P. Giblin, J. P. Griffiths, G. A. C. Jones, I. Farrer, D. A. Ritchie, and T. J. B. M. Janssen, arXiv:1212.4981v1.
${ }^{16}$ F. Battista and P. Samuelsson, Phys. Rev. B 83, 125324 (2011).

${ }^{17}$ V. Kashcheyevs and J. Timoshenko, Phys. Rev. Lett. 109, 216801 (2012).

${ }^{18}$ S. Ol'khovskaya, J. Splettstoesser, M. Moskalets, and M. Büttiker, Phys. Rev. Lett. 101, 166802 (2008).

${ }^{19}$ J. Keeling, I. Klich, and L. S. Levitov, Phys. Rev. Lett. 97, 116403 (2006).

${ }^{20}$ J. Keeling, A. V. Shytov, and L. S. Levitov, Phys. Rev. Lett. 101, 196404 (2008)

${ }^{21}$ J. Zhang, Y. Sherkunov, N. d'Ambrumenil, and B. Muzykantskii, Phys. Rev. B 80, 245308 (2009).

${ }^{22}$ M. Moskalets and M. Büttiker, Phys. Rev. B 83, 035316 (2011).

${ }^{23}$ J. Splettstoesser, M. Moskalets, and M. Büttiker, Phys. Rev. Lett. 103, 076804 (2009).

${ }^{24}$ J. Splettstoesser, P. Samuelsson, M. Moskalets, and M. Büttiker, J. Phys. A: Math. Theor. 43, 354027 (2010).

${ }^{25}$ Y. Sherkunov, N. d'Ambrumenil, P. Samuelsson, and M. Büttiker, Phys. Rev. B 85, 081108 (2012).

${ }^{26}$ C. H. Bennett and D. P. DiVincenzo, Nature (London) 404, 247 (2000).

${ }^{27}$ J. Splettstoesser, S. Ol'khovskaya, M. Moskalets, and M. Büttiker, Phys. Rev. B 78, 205110 (2008).

${ }^{28}$ M. Moskalets and M. Büttiker, Phys. Rev. B 80, 081302(R) (2009).

${ }^{29}$ S. Juergens, J. Splettstoesser, and M. Moskalets, Europhys. Lett. 96, 37011 (2011).

${ }^{30}$ L. S. Levitov, H. Lee, and G. B. Lesovik, J. Math. Phys. 37, 4845 (1996).

${ }^{31}$ D. A. Ivanov, H. W. Lee, and L. S. Levitov, Phys. Rev. B 56, 6839 (1997).

${ }^{32}$ J. Dubois, T. Jullien, C. Grenier, P. Degiovanni, P. Roulleau, and D. C. Glattli, arXiv:1212.3921.

${ }^{33}$ J. Dubois, T. Jullien, P. Roulleau, F. Portier, P. Roche, A. Cavanna, Y. Jin, W. Wegscheider, and D. C. Glattli (private communication).

${ }^{34}$ J. Gabelli, G. Fève, J.-M. Berroir, B. Plaçais, A. Cavanna, B. Etienne, Y. Jin, and D. C. Glattli, Science 313, 499 (2006).

${ }^{35}$ F. D. Parmentier, E. Bocquillon, J.-M. Berroir, D. C. Glattli, B. Plaçais, G. Féve, M. Albert, C. Flindt and M. Büttiker, Phys. Rev. B 85, 165438 (2012). 
${ }^{36}$ A. Prêtre, H. Thomas, and M. Büttiker, Phys. Rev. B 54, 8130 (1996).

${ }^{37}$ M. Moskalets, P. Samuelsson, and M. Büttiker, Phys. Rev. Lett. 100, 086601 (2008).

${ }^{38}$ M. Büttiker, H. Thomas, and A. Prêtre, Phys. Lett. A 180, 364 (1993).

${ }^{39}$ K. Sasaoka, T. Yamamoto, and S. Watanabe, Appl. Phys. Lett. 96, 102105 (2010).

${ }^{40}$ G. Haack, M. Moskalets, J. Splettstoesser, and M. Büttiker, Phys. Rev. B 84, 081303(R) (2011).

${ }^{41}$ G. Haack, Ph.D. thesis, University of Geneva, 2012 [http://archive-ouverte.unige.ch/unige:22610].

${ }^{42}$ M. Büttiker, Phys. Rev. B 46, 12485 (1992).

${ }^{43}$ R. Liu, B. Odom, Y. Yamamoto, and S. Tarucha, Nature (London) 391, 263 (1998).

${ }^{44}$ Y. M. Blanter and M. Büttiker, Phys. Rep. 336, 1 (2000).

${ }^{45}$ G. Fève, P. Degiovanni, and T. Jolicoeur, Phys. Rev. B 77, 035308 (2008).

${ }^{46}$ M. Moskalets and M. Büttiker, Phys. Rev. B 66, 205320 (2002).

${ }^{47}$ C. K. Hong, Z. Y. Ou, and L. Mandel, Phys. Rev. Lett. 59, 2044 (1987).

${ }^{48}$ T. Jonckheere, J. Rech, C. Wahl, and T. Martin, Phys. Rev. B 86, 125425 (2012).

${ }^{49}$ R. Loudon, in Disorder in Condensed Matter Physics, edited by J. A. Blackman and J. Taguena (Clarendon, Oxford, 1991), p. 441.
${ }^{50}$ E. Bocquillon, V. Freulon, J.-M. Berroir, P. Degiovanni, B. Plaçais, A. Cavanna, Y. Jin, and G. Fève, Science 339, 1054 (2013).

${ }^{51}$ S. E. Nigg, R. Lopez, and M. Büttiker, Phys. Rev. Lett. 97, 206804 (2006).

${ }^{52}$ C. Petitjean, D. Waltner, J. Kuipers, I. Adagideli, and K. Richter, Phys. Rev. B 80, 115310 (2009).

${ }^{53}$ C. Mora and K. Le Hur, Nat. Phys. 6, 697 (2010).

${ }^{54}$ O. Kashuba, H. Schoeller, and J. Splettstoesser, Europhys. Lett. 98, 57003 (2012).

${ }^{55}$ S. E. Nigg and M. Büttiker, Phys. Rev. B 77, 085312 (2008).

${ }^{56}$ J. E. Avron, A. Elgart, G. M. Graf, and L. Sadun, Phys. Rev. Lett. 87, 236601 (2001); J. Math. Phys. 43, 3415 (2002).

${ }^{57}$ M. Kataoka, J. D. Fletcher, P. See, S. P. Giblin, T. J. B. M. Janssen, J. P. Griffiths, G. A. C. Jones, I. Farrer, and D. A. Ritchie, Phys. Rev. Lett. 106, 126801 (2011).

${ }^{58}$ F. Battista and P. Samuelsson, Phys. Rev. B 85, 075428 (2012).

${ }^{59}$ L. Arrachea, M. Moskalets, and L. Martin-Moreno, Phys. Rev. B 75, 245420 (2007).

${ }^{60}$ L. Arrachea and B. Rizzo, arXiv:1212.3181.

${ }^{61}$ M. Moskalets and M. Büttiker, Phys. Rev. B 78, 035301 (2008).

${ }^{62}$ M. Büttiker, H. Thomas, and A. Prêre, Z. Phys. B 94, 133 (1994).

${ }^{63}$ P. W. Brouwer, Phys. Rev. B 58, 10135 (1998). 